\title{
Dynamic Networked Coverage of Time-Varying Environments in the Presence of Fading Communication Channels
}

\author{
ALIREZA GHAFFARKHAH, Google \\ YASAMIN MOSTOFI, University of California, Santa Barbara
}

\begin{abstract}
In this paper, we study the problem of dynamic coverage of a set of points of interest (POIs) in a time-varying environment. We consider the scenario where a physical quantity is constantly growing at certain rates at the POIs. A number of mobile agents are then deployed to periodically cover (sense or service) the POIs and keep the physical quantity under control bounded at all the POIs. We assume a communication-constrained operation, where the mobile agents need to communicate to a fixed remote station over realistic wireless links to complete their coverage task. We then propose novel mixed-integer linear programs (MILPs) to design periodic trajectories and TX power policies for the mobile agents that minimize the total energy (the summation of motion and communication energy) consumption of the mobile agents in each period, while 1) guaranteeing the boundedness of the quantity of interest at all the POIs, and 2) meeting the constraints on the connectivity of the mobile agents, the frequency of covering the POIs, and the total energy budget of the mobile agents. We furthermore provide a probabilistic analysis of the problem. Our results show the superior performance of the proposed framework for dynamic coverage in realistic fading environments.
\end{abstract}

Categories and Subject Descriptors: C.2.0 [Computer-Communication Networks]: General; C.2.4 [Computer-Communication Networks]: Distributed Systems

General Terms: Design, Algorithms, Performance, Theory

Additional Key Words and Phrases: Wireless sensor networks, Dynamic coverage of time-varying environments, Communication-aware trajectory planning, Power adaptation, Channel learning, Energy conservation

\section{INTRODUCTION}

Deployment of a group of mobile agents to dynamically cover a spatially large environment has a broad range of applications in robotics and mobile sensor networks [Smith et al. 2011; Smith et al. 2012; Grocholsky et al. 2006; Wang and Hussein 2010]. In a spatially-large environment, there exist a number of points of interest (POIs) that cannot be fully covered by any static configuration of the mobile agents, possibly due to the small effective ranges of their onboard sensors/actuators compared to the size of the environment. In the dynamic coverage problem, we are then interested in planning the motion of the mobile agents such that they can cover all the POIs in a spatially-large environment. This translates to planning the motion of the mobile agents to minimize/maximize/bound a quantity of interest at the POIs.

A small part of this work appeared in the 2nd IEEE Globecom International Workshop on Wireless Networking for Unmanned Autonomous Vehicles (Wi-UAV'11) [Ghaffarkhah et al. 2011].

This work is supported by US National Science Foundation CAREER award \#0846483 and ARO CTA MAST Project W911NF-08-2-0004.

Authors' address: Alireza Ghaffarkhah, Google Inc, 1600 Amphitheatre Parkway, Mountain View, CA email: alinem@gmail.com; Yasamin Mostofi, Department of Electrical and Computer Engineering, University of California, Santa Barbara, CA email: ymostofi@ece.ucsb.edu.

Permission to make digital or hard copies of part or all of this work for personal or classroom use is granted without fee provided that copies are not made or distributed for profit or commercial advantage and that copies show this notice on the first page or initial screen of a display along with the full citation. Copyrights for components of this work owned by others than ACM must be honored. Abstracting with credit is permitted. To copy otherwise, to republish, to post on servers, to redistribute to lists, or to use any component of this work in other works requires prior specific permission and/or a fee. Permissions may be requested from Publications Dept., ACM, Inc., 2 Penn Plaza, Suite 701, New York, NY 10121-0701 USA, fax +1 (212) 869-0481, or permissions@acm.org.

(C) YYYY ACM 1550-4859/YYYY/01-ARTA $\$ 10.00$

DOI 10.1145/0000000.0000000 http://doi.acm.org/10.1145/0000000.0000000 


\begin{tabular}{|c|c|c|c|}
\hline$m$ & Number of POIs & $P_{\ell, s, k}$ & $\begin{array}{l}\text { Power loss when the } k \text { th agent stops at } \\
\text { a POI for sensing/servicing }\end{array}$ \\
\hline$n$ & Number of mobile agents & $P_{\ell, m, k}$ & Power loss when the $k$ th agent moves \\
\hline $\mathcal{W}$ & Workspace (a subset of $\mathbb{R}^{2}$ ) & $P_{\mathrm{TX}, \max , k}$ & Max TX power of the $k$ th agent \\
\hline $\mathcal{Q}$ & Set of the positions of the POIs & $\mathcal{E}_{\max , k}$ & $\begin{array}{l}\text { Total energy budget of the } k \text { th agent } \\
\text { in each period }\end{array}$ \\
\hline $\mathcal{V}$ & Set of indices of the POIs & $T_{\max }$ & Max allowable period for each agent \\
\hline $\mathcal{V}_{k}$ & $\begin{array}{l}\text { Set of indices of the POIs assigned to } \\
\text { the } k \text { th agent }\left(\bigcup_{k=1}^{n} \mathcal{V}_{k}=\mathcal{V}\right)\end{array}$ & $t_{c}$ & $\begin{array}{l}\text { Communication time at each pre- } \\
\text { selected communication point }\end{array}$ \\
\hline$q_{i}$ & Position of the $i$ th POI & $P_{\mathrm{TX}, i, k}$ & $\begin{array}{l}\text { TX power of the } k \text { th agent at the } i \text { th } \\
\text { POI in the comm-intensive case }\end{array}$ \\
\hline$t_{i, k}$ & $\begin{array}{l}\text { Stop time of the } k \text { th agent at the } i \text { th } \\
\text { POI }\end{array}$ & $P_{\mathrm{TX}, k}$ & $\begin{array}{l}\text { TX power of the } k \text { th agent at its comm } \\
\text { point in the comm-efficient case }\end{array}$ \\
\hline$\xi_{k}(t)$ & Position of the $k$ th agent at time $t$ & $\xi_{\mathrm{TX}, k}$ & $\begin{array}{l}\text { Comm point of the } k \text { th agent in the } \\
\text { comm-efficient case }\end{array}$ \\
\hline$\Psi_{i}(t)$ & Quantity of interest at the $i$ th POI & $v_{\max }$ & Max velocity of the $k$ th agent \\
\hline$\rho_{i}$ & $\begin{array}{l}\text { Rate at which } \Psi_{i}(t) \text { increases linearly } \\
\text { at the } i \text { th POI }\end{array}$ & $\mathcal{H}_{k}$ & $\begin{array}{l}\text { Hamiltonian cycle on the set of POIs } \\
\text { assigned to the } k \text { th agent }\end{array}$ \\
\hline$\alpha_{i, k}$ & Service rate of $k$ th agent at the $i$ th POI & $\mathcal{H}_{k}^{*}$ & $\begin{array}{l}\text { Min-length Hamiltonian cycle on the } \\
\text { set of POIs assigned to the } k \text { th agent }\end{array}$ \\
\hline$\lambda_{k}(t)$ & $\begin{array}{l}\text { Binary variable which is } 1 \text { if the } k \text { th } \\
\text { agent is connected to the remote sta- } \\
\text { tion at time } t \text {, and is } 0 \text { otherwise }\end{array}$ & $P_{\mathrm{TX}, \min }(q, \chi)$ & $\begin{array}{l}\text { Min required TX power to guarantee } \\
\text { the probability of connectivity at point } \\
q \text { is no less than } \chi \text { (see Section } 2.1)\end{array}$ \\
\hline$G(q)$ & $\begin{array}{l}\text { Stochastic channel power at position } q \\
\left(G_{\mathrm{dB}}(q) \text { denotes the value of } G(q) \text { in }\right. \\
\text { the } \mathrm{dB} \text { domain })\end{array}$ & $\widetilde{P}_{\mathrm{TX}, \min }(\chi)$ & $\begin{array}{l}\text { Min required TX power to guarantee } \\
\text { the probability of connectivity at a } \\
\text { random point in } \mathcal{W} \text { is no less than } \chi \\
\text { (see Section } 5 \text { ) }\end{array}$ \\
\hline$\hat{G}_{\mathrm{dB}}(q)$ & $\begin{array}{l}\text { Predicted channel power at position } q \\
\text { in the } \mathrm{dB} \text { domain (see Appendix A) }\end{array}$ & $\mathrm{d}\left(\mathcal{H}_{k}\right)$ & Euclidean length of $\mathcal{H}_{k}$ \\
\hline$\sigma^{2}(q)$ & $\begin{array}{l}\text { Variance of channel power prediction } \\
\text { at position } q \text { (see Appendix A) }\end{array}$ & $\theta$ & Path loss parameters (see Appendix A) \\
\hline $\mathrm{SNR}_{\mathrm{TH}}$ & $\begin{array}{l}\text { Min received SNR required for connec- } \\
\text { tivity at the receiver of the remote sta- } \\
\text { tion }\end{array}$ & $\vartheta^{2}, \beta$ & $\begin{array}{l}\text { Variance (power) and decorrelation } \\
\text { distance of the shadowing component } \\
\text { of the channel (see Appendix A) }\end{array}$ \\
\hline$N_{0} B$ & $\begin{array}{l}\text { Power of thermal noise at the receiver } \\
\text { of the remote station ( } B \text { is the total } \\
\text { bandwidth) }\end{array}$ & $\omega^{2}$ & $\begin{array}{l}\text { Variance (power) of the multipath fad- } \\
\text { ing component of the channel (see Ap- } \\
\text { pendix A) }\end{array}$ \\
\hline
\end{tabular}

Table I: List of the main variables used throughout this paper.

In this paper, we consider a networked dynamic coverage problem, an extended version of the dynamic coverage problem where a number of mobile agents, with limited energy budgets and sensing/actuation capabilities, are deployed to cover a set of POIs in a timevarying environment. By a time-varying environment, we refer to an environment where the quantity of interest is time-varying and increasing in time at every POI that is not in the effective range of any mobile agent. By networked, we consider a communicationconstrained scenario, where the mobile agents are required to communicate to a fixed remote station in order to complete their coverage task. Our goal in this paper is then to plan the motion and communication policies of the mobile agents to minimize the total energy (the summation of the motion and communication energy) consumption of the mobile agents, while 1) guaranteeing the boundedness of the quantity of interest at all the POIs, and 2) meeting the constraints on the connectivity of the mobile agents to the remote station, the frequency of covering the POIs, and the total energy budget of the mobile agents. Note that since the quantity of interest is continuously increasing at the POIs, periodic trajectories need to be devised for the mobile agents in order to repeatedly cover the POIs. A schematic of the dynamic coverage problem considered in this paper is shown in Fig. 1.

Several real-world applications can be modeled by a dynamic coverage problem. Next, we provide a number of examples from mobile sensor networks and robotics literature: 


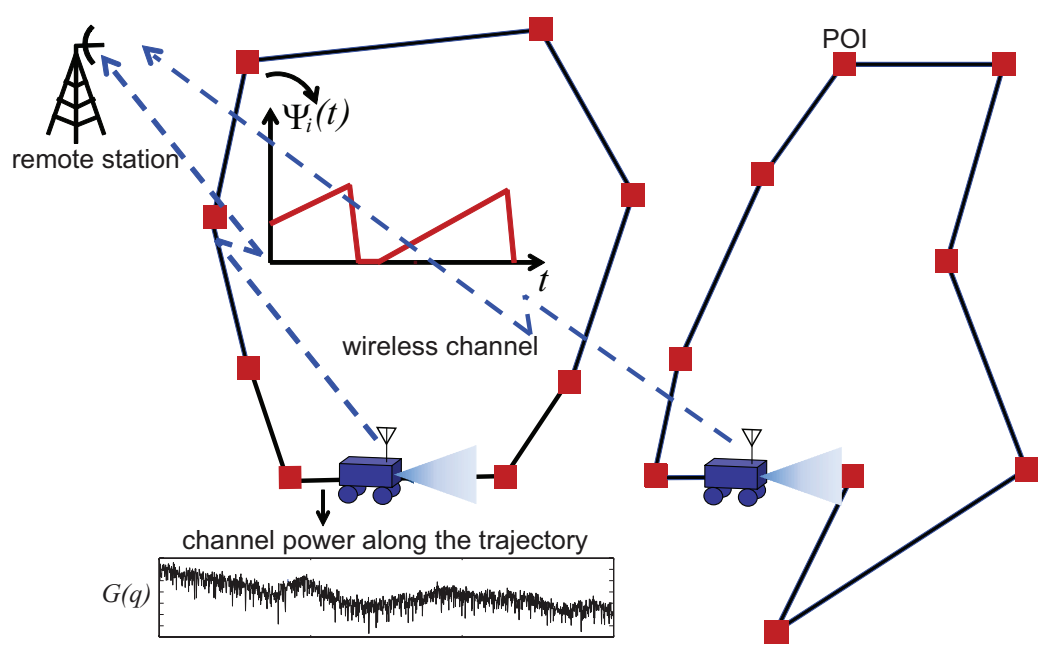

Fig. 1: Dynamic coverage of a time-varying environment using a team of mobile agents. $\Psi_{i}(t)$ is the quantity of interest to keep bounded at the $i$ th POI.

(1) The first example is surveillance and monitoring of a time-varying environment using a team of mobile agents. Here, the remote station is a monitoring station and the quantity of interest that needs to be kept bounded is the uncertainty on the time-varying states of the POIs at the remote station. In this example, a POI is covered if it can be sensed by the onboard sensor of a mobile agent (e.g., a digital camera).

(2) The second example is estimation over wireless communication links. The POIs in this example represent a number of dynamical systems, spatially distributed over the workspace, whose states need to be estimated at a remote station. A number of mobile agents then observe the dynamical systems along periodic trajectories and send their observations to the remote station over wireless communication links. A POI is covered in this examples if its state can be observed by a mobile agent and communicated to the remote station. The quantity of interest to keep bounded for each POI is then the uncertainty of its state estimation (e.g., the estimation error variance) at the remote station. This uncertainty is increasing in time when the POI is not covered by any agent. This problem can be thought of as an extension of the problem of estimating dynamical systems over wireless links, which received considerable attention in recent years [Sinopoli et al. 2004].

(3) The third example is information collection in a time-varying environment, where the POIs represent a number of stationary data loggers that are distributed over a spatiallylarge environment to log time-variations of an environmental feature (e.g. temperature, humidity, radioactive contamination). The information bits (which are increasing in time at each data logger) need to be collected and transmitted to a remote station. A number of mobile agents are then tasked to move along periodic trajectories, collect the information bits from the data loggers, and transmit them to the remote station at positions where they get connected along their trajectories. The quantity of interest to keep bounded in this example is the size of the queue of the data loggers through proper information collection and communication.

In all these examples, communication to the remote station is needed and considering the effect of realistic fading communication channels between the mobile agents and the remote station is considerably important. A communication-aware strategy is then required to co-optimize the information-gathering (local coverage) and information-exchange (com- 
munication) performance of the mobile agents. Next, we explain our communication-aware approach for dynamic coverage of time-varying environments in more details.

We assume a linear ${ }^{1}$ dynamics for the time-variation of the quantity of interest at the POIs and a limited total energy budget for the mobile agents. We also consider the case where the sensing/actuation range of the mobile agents is small such that each agent is required to move to the position of each POI and stop there for some time to sense/service it. Then, we optimize motion (trajectories and stop times) and communication (transmission powers) of the mobile agents to minimize the total energy consumption of the mobile agents in each period, while guaranteeing that the quantity of interest at the POIs remains bounded, and the constraints on the connectivity of the mobile agents, the frequency of covering the POIs, and the total energy budget of the mobile agents are satisfied. To keep our framework general, we consider two variants of the problem: communication-intensive and communication-efficient. Communication-intensive case refers to the case where the mobile agents are required to be connected at all the POIs they visit, in order to send their collected information to the remote station in real-time. Communication-efficient case, on the other hand, refers to the case where the mobile agents are only required to connect to the remote station once along their trajectories, decreasing the communication burden considerably. In both communication-intensive and communication-efficient cases, we show how to optimally find the trajectories of the mobile agents, as well as their stop times and transmission powers, using mixed-integer linear programs (MILPs). The properties of the optimal solutions of the MILPs, as well as their asymptotic properties, are also characterized mathematically. ${ }^{2}$ To the best of our knowledge, this is the first time that dynamic coverage is solved optimally, in the presence of realistic communication channels and under several constraints on the connectivity and total energy consumption of the mobile agents. Also, there is no existing work that mathematically analyzes the dynamic coverage problem, as we do so in this paper.

\subsection{Related Work}

The existing literature related to the dynamic coverage problem of this paper is categorized based on the type of the environment (time-invariant or time-varying) and motion planning approach (analytical or algorithmic). For instance, the exploration strategies of [Wang and Hussein 2010] can be considered dynamic coverage strategies used to cover a time-invariant environment based on analytical motion planning approaches (gradient-based approaches). The algorithmic motion planning approaches of [Choset 2001; Acar and Choset 2002; Acar et al. 2006] can also be used for dynamic coverage of a time-invariant environment. In these works, the authors determine the paths that pass through a set of points or cells in a known [Choset 2001] or unknown [Acar and Choset 2002] environment. Their proposed approaches involve 1) cellular decomposition (for known environments) or Morse decomposition (for unknown environments), and 2) devising heuristic and exact algorithms to achieve coverage. In their more recent work in [Acar et al. 2006], the authors also extend their algorithmic approach to the case of sensing ranges that go beyond the size of the robot. These works, however, do not consider planning periodic trajectories for dynamic coverage of time-varying environments. Furthermore, none of these works consider realistic communication and energy constraints when planning the motion of the mobile agents.

In terms of the class of the trajectories considered, the proposed approaches of this paper are related to current literature on sweep coverage and patrolling [Li et al. 2011; Chevaleyre 2004; Machado et al. 2003; Agmon et al. 2011; Elmaliach et al. 2009] and persistent monitoring [Hokayem et al. 2007; Smith et al. 2011], where periodic trajectories for the mobile

\footnotetext{
${ }^{1}$ While the dynamics of the quantity of interest in the aforementioned problems could be nonlinear, a linear approximation may be a close enough approximation depending on the system parameters.

${ }^{2}$ It should be noted that given the constraints of the dynamic coverage problem, a feasible solution may not exit. Our proposed MILPs find the optimal feasible solution if such a policy exists.
} 
agents are planned to repeatedly cover a set of POIs in the environment. The approaches of [Li et al. 2011; Chevaleyre 2004; Machado et al. 2003; Agmon et al. 2011; Elmaliach et al. 2009; Hokayem et al. 2007] are based on designing heuristic near-optimal algorithms for covering the POIs (under a constraint on the frequency of visiting the points or by maximizing the frequency of the visits). The authors, however, do not consider a time-varying environment and realistic communication and energy constraints. The authors in [Smith et al. 2011] propose a trajectory planning algorithm, based on a constrained version of the Bellman-Ford algorithm, to persistently visit a set of cells in a discretized version of the environment. Their goal is to maximize a reward function and meet the constraint on the maximum allowable time for an agent to complete a cycle, without considering the communication and energy issues. The formal definition of a time-varying environment that we utilize in this paper is first presented in [Smith et al. 2012], where the authors introduce the dynamics of the quantity of interest at the POIs. In order to stabilize the dynamic coverage task, they then propose strategies to adapt the velocities of the mobile agents along predefined periodic trajectories. Similarly, no communication or energy constraint is considered in [Smith et al. 2012]. In this paper, we extend the previous work on multi-agent coverage to a time-varying environment and in the presence of communication, time and energy constraints. More specifically, we consider a generalized version of the linear dynamical model of [Smith et al. 2012] to capture the time variations of the quantity of interest in the presence of realistic fading channels. We then propose optimal trajectories, stop times and transmission powers for the mobile agents to minimize the total energy consumption of the mobile agents in each period, while guaranteeing that the quantity of interest at the POIs remains bounded, and the constraints on the connectivity of the mobile agents, the frequency of covering the POIs, and the total energy budget of the mobile agents are satisfied. Our proposed approach enables networked multi-agent dynamic coverage in realistic communication settings, which is not possible using the current methods.

The rest of the paper is organized as follows. In Section 2, we introduce the dynamical models of the quantity of interest at the POIs, as well as the connectivity and energy consumption models of the mobile agents. The dynamic coverage problems in the communication-intensive and communication-efficient cases are formulated and solved using MILPs in Sections 3 and 4. Probabilistic analysis of the dynamic coverage problem is studied in Section 5. We present our simulation results in Section 6, followed by conclusions in Section 7. A list of main variable used throughout the paper is also provided in Table I.

\section{SYSTEM MODEL}

Consider an obstacle-free ${ }^{3}$ workspace $\mathcal{W} \subset \mathbb{R}^{2}$ that contains a set of $m$ POIs $\mathcal{Q}=$ $\left\{q_{1}, \cdots, q_{m}\right\}$. Let $\Psi_{i}(t)$, for $i=1, \cdots, m$, represent the quantity of interest that need to be controlled at the $i$ th POI. We assume a time-varying workspace, where $\Psi_{i}(t)$ increases at a certain rate as long as the $i$ th POI is not being covered ${ }^{4}$ by any mobile agent. Then, as soon as the POI is covered by a mobile agent, $\Psi_{i}(t)$ decreases at a rate that depends on the onboard capabilities of the mobile agent. In order to keep $\Psi_{i}(t)$ bounded at all the POIs, we use a team of $n$ mobile agents. Each mobile agent is assigned to a nonempty subset of the POIs. A closed periodic trajectory is then planned for each agent to repeatedly cover every point in this subset.

\footnotetext{
${ }^{3}$ Note that the results of Sections 3 and 4 are readily applicable to the case where there exists some obstacles in the workspace as well. In this case it is sufficient to replace the Euclidean distance between the POIs with the length of the obstacle-free path between them.

${ }^{4}$ The term "covered" in this paper refers to being sensed/serviced by the onboard sensor/actuator of a mobile agent.
} 
Let $\mathcal{V}=\{1, \cdots, m\}$ denote the set of the indices of the POIs. Also, let $\mathcal{V}_{k}$, for $k=1, \cdots, n$, represent the nonempty subset of $\mathcal{V}$ assigned to the $k$ th agent. In this paper, we consider the following assumptions:

Assumption 2.1. The sets $\left\{\mathcal{V}_{k}\right\}_{k=1}^{n}$ define a partition of $\mathcal{V}$, i.e., $\bigcup_{k=1}^{n} \mathcal{V}_{k}=\mathcal{V}$ and $\mathcal{V}_{k_{1}} \cap \mathcal{V}_{k_{2}}=\emptyset$, for $k_{1} \neq k_{2}$. Therefore, each POI is assigned to one agent only.

Assumption 2.2. The effective ranges of the onboard sensors/actuators of the mobile agents are negligible, compared to the size of the workspace. Therefore, in order to cover each POI, each agent is required to physically move to the position of the POI.

These assumptions imply that 1 ) the optimal trajectory for the $k$ th agent, without loss of generality, is a Hamiltonian cycle on the set of POIs in $\mathcal{V}_{k}$ and 2) due to negligible effective ranges, each agent requires to stop for a limited time at each POI to sense/service it. In this paper, we adopt an extended version of the linear model proposed in [Smith et al. 2012] for the dynamics of $\Psi_{i}(t)$ :

$$
\begin{aligned}
& \Psi_{i}(t)=\Phi_{i}\left(\tau_{k}(t)\right)+\rho_{i}\left[t-\tau_{k}(t)\right], \\
& \dot{\Phi}_{i}(t)=\mathrm{I}\left(\Phi_{i}(t) \geq 0\right)\left[\rho_{i}-\mathrm{I}\left(\xi_{k}(t)=q_{i}\right) \alpha_{i, k}\right], \quad \forall i \in \mathcal{V}_{k}, k=1, \cdots, n,
\end{aligned}
$$

where $\mathrm{I}($.$) denotes the indicator function, \xi_{k}(t)$ is the position of the $k$ th mobile agent at time $t, \lambda_{k}(t)$ is a binary value which is one if the $k$ th agent is connected to the remote station at time $t$ along its trajectory and zero otherwise, and $\tau_{k}(t) \triangleq \max \left\{0 \leq \tau \leq t \mid \lambda_{k}(\tau)=1\right\}$ specifies the last time the $k$ th agent has been connected to the remote station up to time $t$. Furthermore, $\rho_{i}$ determines the constant rate at which $\Psi_{i}(t)$ increases while it is not being covered by any mobile agent, $\alpha_{i, k}$ represents the constant service rate of the $k$ th mobile agent at the $i$ th POI and $\Phi_{i}(t)$ is an auxiliary quantity.

The dynamical model of (1) implies that $\Psi_{i}(t)$, for $i \in \mathcal{V}_{k}$, increases with rate $\rho_{i}$ while the $k$ th mobile agent is not connected to the remote station. Then, whenever the mobile agent gets connected and communicates to the remote station, $\Psi_{i}(t)$ becomes equal to $\Phi_{i}(t)$, which can be treated as the local version of $\Psi_{i}(t)$ at the $k^{\text {th }}$ mobile agent. $\Phi_{i}(t)$ itself increases with rate $\rho_{i}$ while the $k$ th mobile agent is not at the $i$ th POI, and decreases with rate $\alpha_{i, k}-\rho_{i}$ otherwise.

Depending on how often the mobile agents are required to communicate along their trajectories, we consider two cases: communication-intensive and communication-efficient. In the communication-intensive case, the mobile agent $k$ is required to be connected and communicate to the remote station at all the POIs in $\mathcal{V}_{k}$. This case is suitable for the scenarios where the remote station requires a constant update on the states of the POIs or communication to the remote station is needed for the operation of the coverage process. In the communication-efficient case, on the other hand, connectivity at all the POIs is not required. Each mobile agent $k$ covers the POIs in $\mathcal{V}_{k}$ and completes its coverage task by communicating to the remote station at one pre-selected position along its trajectory, reducing the communication burden considerably. At this position, the mobile agent informs the remote station of the states of all the POIs it has covered in one period. We next continue with the connectivity and energy consumption models of the mobile agents.

\subsection{Connectivity Model of the Mobile Agents}

The binary value $\lambda_{k}(t)$ used in (1) is a function of the signal-to-noise ratio (SNR) of the channel between the $k$ th agent and the remote station at time $t$ along its trajectory [Goldsmith 2005]. It can be shown that in a realistic communication setting and in the presence of a packet dropping receiver at the remote station, $\lambda_{k}(t)$ is given as follows [Goldsmith 
2005; Ghaffarkhah and Mostofi 2011]:

$$
\lambda_{k}(t)=\left\{\begin{array}{l}
1, \frac{P_{\mathrm{TX}, k}(t) G_{k}(t)}{N_{0} B} \geq \mathrm{SNR}_{\mathrm{TH}}, \quad k=1, \cdots, n, \\
0, \quad \text { otherwise, }
\end{array}\right.
$$

where $P_{\mathrm{TX}, k}(t)$ is the transmission power of the $k$ th agent at time $t$ along its trajectory, $G_{k}(t)$ determines the instantaneous channel power in transmission from the $k$ th agent to the remote station at time $t$ along its trajectory, $N_{0} / 2$ is the power spectral density (PSD) of the receiver noise, and $B$ is the channel bandwidth. Also, $\mathrm{SNR}_{\mathrm{TH}}$ denotes the packet dropping threshold of the receiver of the remote station, which depends on the quality of decoding at the remote station [Goldsmith 2005; Son et al. 2006; Ghaffarkhah and Mostofi 2011]. The instantaneous channel power $G_{k}(t)$ is a function of the position of the $k$ th agent at time $t: G_{k}(t)=G\left(\xi_{k}(t)\right)$, where $G(q)$, for $q \in \mathcal{W}$, denotes the 2D map of channel power in the workspace. In practical applications, the channel power $G(q)$ is either unknown or known only at a small number of positions, different from the positions of the POIs. In such cases, $G(q)$ is best modeled probabilistically. In Appendix A, we briefly introduce a multi-scale probabilistic model of wireless channels and present our previously-proposed probabilistic channel assessment framework. This framework enables prediction of the distribution of $G(q)$ at unvisited locations, conditioned on a small number of a priori channel power measurements. In the rest of this paper, we use this framework to probabilistically assess the channel along the trajectory of the mobile agents and find conditions on the transmission powers to increase the probability of connectivity at all the POIs (in the communicationintensive case) or at one pre-selected communication point along the trajectory of each agent (in the communication-efficient case). ${ }^{5}$

Note that in the communication-intensive case, each mobile agent needs to be connected and communicate to the remote station at all its assigned POIs. Let $P_{\mathrm{TX}, i, k}$, for $i \in \mathcal{V}_{k}$, denote the transmission power of the $k$ th mobile agent at the $i$ th POI in the communicationintensive case. Also, let $t_{c}$ denote a fixed communication time assigned for communicating to the remote station. We assume that $t_{c}$ is small enough such that sending/receiving the packets at each POI can be finished while the mobile agent is stopped at the POI (or still very close to it). This assumption facilitates mathematical derivations by ensuring that the channel power remains stationary while communicating at each POI. ${ }^{6}$ Then, in the communication-intensive case we have $P_{\mathrm{TX}, k}(t)=P_{\mathrm{TX}, i, k}$ for a time period of length $t_{c}$ at (or close to) the $i$ th POI, and $P_{\mathrm{TX}, k}(t)=0$ otherwise. In the communication-efficient case, on the other hand, the mobile agents communicate to the remote station at one pre-selected point along their trajectories. Let $\xi_{\mathrm{TX}, k}$ and $P_{\mathrm{TX}, k}$ denote the communication point of the $k$ th agent and its fixed transmission power at this point in the communication efficient case, respectively. Similar to the communication-intensive case, we then have $P_{\mathrm{TX}, k}(t)=P_{\mathrm{TX}, k}$ for a time period of length $t_{c}$ at (or close to) position $\xi_{\mathrm{TX}, k}$, and $P_{\mathrm{TX}, k}(t)=0$ otherwise. We find the optimal values of $P_{\mathrm{TX}, i, k}$, for $i \in \mathcal{V}_{k}$, in Section 3 and the optimal values of $\xi_{\mathrm{TX}, k}$ and $P_{\mathrm{TX}, k}$ in Section 4.

\subsection{Energy Consumption Model of the Mobile Agents}

The total energy consumed by a mobile agent in one period is the summation of its motion energy and its communication energy. The motion energy is the time integral of the motion

\footnotetext{
${ }^{5}$ In case the mobile agents are able to measure the channel, $G(q)$ is assessed probabilistically to initially plan the coverage policy. Then, after the first period $G(q)$ will be known at the POIs.

${ }^{6}$ This is a fair assumption as the transmission rates when connected are usually large. For instance in IEEE $802.11 \mathrm{~g}$ the maximum achievable rate is $54 \mathrm{Mbit} / \mathrm{s}$, which implies that sending/receiving a large packet of data in case of a good channel does not take more than a couple of seconds. Note that the results of this paper can also be extended to the case where transmission occurs throughout the trajectory as well, i.e. the case that $t_{c}$ is bigger than the sensing time at a POI.
} 
power, which itself is a function of the velocity and power loss of the mobile agent. We adopt the following model for the motion power of the $k$ th agent [Mei et al. 2006; 2005]:

$$
P_{m, k}(t)=\left\{\begin{array}{l}
P_{\ell, s, k}, \\
P_{\ell, m, k}+w_{k} v_{k}, \quad \xi_{k}(t)=q_{i}, \text { for some } i \in \mathcal{V}_{k},
\end{array}\right.
$$

where $v_{k}$ and $P_{\ell, m, k}$ are the velocity and the power loss of the $k$ th agent while moving from one POI to another, $P_{\ell, s, k}$ denotes its power loss while stopping at one of the POIs, and $w_{k}$ is a constant that depends on the dynamics of the $k$ th agent. Note that we include all the constant power losses (i.e., motion, computation and actuation losses) in $P_{\ell, m, k}$ and $P_{\ell, s, k}$. Therefore, generally $P_{\ell, m, k}$ is different from (typically smaller than) $P_{\ell, s, k}$. Another note is that, without loss of generality, the velocity of the each mobile agent is assumed constant. The reason is that both sensing/actuation and communication happen either at the positions of the POIs or at one point along the trajectory of the mobile agents. Therefore, adaptation of speed is not required. In fact the optimal velocity for each mobile agent is the maximum possible velocity, as shown in the next section.

Let $\mathcal{H}_{k}$ denote the Hamiltonian cycle defined on the set of POIs in $\mathcal{V}_{k}$, with $\mathrm{d}\left(\mathcal{H}_{k}\right)$ denoting its total Euclidean length. Also, let $t_{i, k}$, for $i \in \mathcal{V}_{k}$, denote the stop time of the $k$ th mobile agent at the $i$ th POI. The motion energy consumed in one period by the $k$ th agent, in both communication-intensive and communication-efficient cases, is then calculated as follows:

$$
\mathcal{E}_{m, k}=P_{\ell, s, k} \sum_{i \in \mathcal{V}_{k}} t_{i, k}+\left(\frac{P_{\ell, m, k}}{v_{k}}+w_{k}\right) \mathrm{d}\left(\mathcal{H}_{k}\right)
$$

The communication energy, on the other hand, is consumed when a mobile agent transmits data to the remote station. Based on the connectivity model of the mobile agents discussed in the previous section, the communication energy consumed in one period by the $k$ th agent in the communication-intensive case becomes:

$$
\mathcal{E}_{\mathrm{TX}, k}=t_{c} \sum_{i \in \mathcal{V}_{k}} P_{\mathrm{TX}, i, k} .
$$

Similarly, in the communication-efficient case, we have the following for communication energy consumed in one period by the $k$ th agent:

$$
\mathcal{E}_{\mathrm{TX}, k}=t_{c} P_{\mathrm{TX}, k} .
$$

Finally, the total energy consumed by the $k$ th agent in one period is given as $\mathcal{E}_{k}=\mathcal{E}_{m, k}+$ $\mathcal{E}_{\mathrm{TX}, k}$. In Sections 3 and 4 , we consider a constraint on the total energy consumption of the mobile agents in one period, when finding the optimal dynamic coverage policies using the proposed MILPs.

\section{DYNAMIC COVERAGE OF TIME-VARYING ENVIRONMENTS IN THE COMMUNICATION-INTENSIVE CASE}

In this section, show how to find optimal feasible dynamic coverage policies for a team of mobile agents in the communication-intensive case using an MILP. Based on the system models presented in Section 2, a dynamic coverage policy in the communication-intensive case, which is a tuple of all the design variables, is defined as follows:

Definition 3.1. A dynamic coverage policy for the $k$ th mobile agent in the communication-intensive case is a tuple $\mathcal{P}_{k}=\left(\mathcal{V}_{k}, \mathcal{H}_{k}, v_{k},\left\{P_{\mathrm{TX}, i, k}\right\}_{i \in \mathcal{V}_{k}},\left\{t_{\mathrm{TX}, i, k}\right\}_{i \in \mathcal{V}_{k}}\right)$. The overall dynamic coverage policy to find is then the tuple $\mathcal{P}=\left(\mathcal{P}_{1}, \cdots, \mathcal{P}_{n}\right)$.

The following lemma gives a necessary and sufficient condition for $\mathcal{P}$ to stabilize the dynamic coverage task: 
LEMMA 3.1. Under the assumption of periodic motion and communication (the same motion and communication policies in each period), a feasible dynamic coverage policy $\mathcal{P}$ is stabilizing, i.e., it stabilizes the dynamic coverage task if 1) it guarantees that the mobile agents are connected at least once along their trajectories, and 2) there exist non-negative stability margins $\Delta_{1}, \cdots, \Delta_{n}$ such that

$$
\alpha_{i, k} t_{i, k}-\rho_{i}\left(\sum_{i \in \mathcal{V}_{k}} t_{i, k}+\frac{\mathrm{d}\left(\mathcal{H}_{k}\right)}{v_{k}}\right) \geq \Delta_{k}, \quad \forall i \in \mathcal{V}_{k}, k=1, \cdots, n .
$$

Proof. A dynamic coverage task is stable, in both communication-intensive and communication-efficient cases, if there exists a finite $\bar{\Psi}$, independent of the initial conditions, such that $\max _{1 \leq i \leq m} \sup _{t \geq 0} \Psi_{i}(t) \leq \bar{\Psi}$. In case the mobile agents are connected at least once along their trajectories, this holds if $\Phi_{i}\left(t+T_{k}\right) \leq \Phi_{i}(t)$, for $i \in \mathcal{V}_{k}$ and $k=1, \cdots, n$, where $T_{k}$ is the period of the $k$ th mobile agent. Based on the dynamical model (1), we have $\Phi_{i}\left(t+T_{k}\right)-\Phi_{i}(t)=\rho_{i} T_{k}-\alpha_{i, k} t_{i, k}$. Furthermore, we have $T_{k}=\sum_{i \in \mathcal{V}_{k}} t_{i, k}+\frac{\mathrm{d}\left(\mathcal{H}_{k}\right)}{v_{k}}$. By substituting $T_{k}$, we obtain the conditions of (7) for stability. Note that these conditions are valid in both communication-intensive and communication-efficient cases.

Based on Lemma 3.1, in order to stabilize the dynamic coverage it is necessary to ensure that each mobile agent is connected at least once along its trajectory. A feasible dynamic coverage policy in the communication-intensive case, however, puts more constraints on the connectivity of the mobile agents and require each mobile agent to be connected at all its assigned POIs. This is to guarantee that the remote station is updated on the states of the POIs as frequently as possible. A feasible policy also satisfies the constraints on stability, the frequency of covering the POIs, the total energy budget, and the maximum transmission power and velocity of the mobile agents. Mathematically, a dynamic coverage policy $\mathcal{P}$ is feasible in the communication-intensive case if the following conditions hold:

1) $\alpha_{i, k} t_{i, k}-\rho_{i}\left(\sum_{i \in \mathcal{V}_{k}} t_{i, k}+\frac{\mathrm{d}\left(\mathcal{H}_{k}\right)}{v_{k}}\right) \geq \Delta_{k}, \quad \forall i \in \mathcal{V}_{k}, k=1, \cdots, n$,

2) $\sum_{i \in \mathcal{V}_{k}} t_{i, k}+\frac{\mathrm{d}\left(\mathcal{H}_{k}\right)}{v_{k}} \leq T_{\max }, \quad k=1, \cdots, n$,

3) $P_{\ell, s, k} \sum_{i \in \mathcal{V}_{k}} t_{i, k}+\left(\frac{P_{\ell, m, k}}{v_{k}}+w_{k}\right) \mathrm{d}\left(\mathcal{H}_{k}\right)+t_{c} \sum_{i \in \mathcal{V}_{k}} P_{\mathrm{TX}, i, k} \leq \mathcal{E}_{\max , k}, \quad k=1, \cdots, n$,

4) $P_{\mathrm{TX}, \max , k} \geq P_{\mathrm{TX}, i, k} \geq \frac{\mathrm{SNR}_{\mathrm{TH}} N_{0} B}{G\left(q_{i}\right)}, \quad \forall i \in \mathcal{V}_{k}, k=1, \cdots, n$,

5) $0 \leq v_{k} \leq v_{\max , k}, \quad k=1, \cdots, n$,

where $T_{\max }$ is the maximum acceptable period for covering all the POIs and $\mathcal{E}_{\max , k}$, $P_{\mathrm{TX}, \max , k}$ and $v_{\max , k}$ are the total energy budget, maximum possible transmission power and maximum velocity of the $k$ th agent. Note that $\frac{\operatorname{SNR}_{\mathrm{TH}} N_{0} B}{G\left(q_{i}\right)}$ is the minimum transmission power required for connectivity at the $i$ th POI.

In case of stochastic wireless channels, $G\left(q_{i}\right)$ is not known and is estimated probabilistically as explained in Appendix A. Then, the constraints of (8) cannot be guaranteed deterministically, since the conditions depend on the stochastic channel powers $G\left(q_{i}\right)$. In such cases, the feasibility can only be guaranteed probabilistically. It is then desired to find deterministic conditions on a policy $\mathcal{P}$ that guarantee feasibility with a probability larger than a given threshold. This is, however, very challenging as the set of feasible $\mathcal{P}$ in (8) is a complex function of the channel powers. To simplify the problem, we use a sub-optimal approach 
from the stochastic programming literature [Shapiro et al. 2009]. The idea is to replace any constraint in (8), that is directly a function of the main random variables $\left(G\left(q_{i}\right)\right.$ in our case), with its chance constraint. A chance constraint is simply a constraint that guarantees that the probability of meeting the stochastic constraint is larger than a given $\chi$, for $0.5<\chi<1$, while assuming that all the other optimization variables are deterministic [Shapiro et al. 2009]. Following this approach, in order to account for stochastic channel powers, it is sufficient to replace $P_{\mathrm{TX}, i, k} \geq \frac{\mathrm{SNR}_{\mathrm{TH}} N_{0} B}{G\left(q_{i}\right)}$ in (8) with $\mathbb{P}\left\{\frac{P_{\mathrm{TX}, i, k} G\left(q_{i}\right)}{N_{0} B} \geq \mathrm{SNR}_{\mathrm{TH}}\right\} \geq \chi$, for a large $0.5<\chi<1$.

To calculate $\mathbb{P}\left\{\frac{P_{\mathrm{TX}, i, k} G\left(q_{i}\right)}{N_{0} B} \geq \mathrm{SNR}_{\mathrm{TH}}\right\}$ we use the channel assessment framework of Appendix A. There, we show that, based on a set of a priori channel power measurements in $\mathcal{W}$, the conditional distribution of $G(q)$ in the $\mathrm{dB}$ domain can be estimated by a Gaussian pdf with mean $\hat{G}_{\mathrm{dB}}(q)$ and variance $\sigma^{2}(q)$, for any $q \in \mathcal{W}$. The exact formulations of $\hat{G}_{\mathrm{dB}}(q)$ and $\sigma^{2}(q)$ as functions of $q$ can be found in Appendix A. We then have

$$
\mathbb{P}\left\{\frac{P_{\mathrm{TX}, i, k} G\left(q_{i}\right)}{N_{0} B} \geq \mathrm{SNR}_{\mathrm{TH}}\right\}=Q\left(\frac{10 \log _{10}\left(\frac{\mathrm{SNR}_{\mathrm{TH}} N_{0} B}{P_{\mathrm{TX}, i, k}}\right)-\hat{G}_{\mathrm{dB}}\left(q_{i}\right)}{\sigma\left(q_{i}\right)}\right),
$$

where $Q(x)=\frac{1}{\sqrt{2 \pi}} \int_{x}^{\infty} e^{-x^{2} / 2} d x$ is the tail probability of Gaussian distribution. After some straightforward calculations, we can find the necessary and sufficient condition for $\mathbb{P}\left\{\frac{P_{\mathrm{TX}, i, k} G\left(q_{i}\right)}{N_{0} B} \geq \mathrm{SNR}_{\mathrm{TH}}\right\} \geq \chi$ as follows:

$$
P_{\mathrm{TX}, i, k} \geq 10^{-\frac{\sigma\left(q_{i}\right) Q^{-1}(\chi)+\hat{G}_{\mathrm{dB}}\left(q_{i}\right)}{10}} \operatorname{SNR}_{\mathrm{TH}} N_{0} B=P_{\mathrm{TX}, \min }\left(q_{i}, \chi\right), \quad \forall i \in \mathcal{V}_{k}, k=1, \cdots, n,
$$

where we defined $P_{\mathrm{TX}, \min }(q, \chi) \triangleq 10^{-\frac{\sigma(q) Q^{-1}(\chi)+\hat{G}_{\mathrm{dB}}(q)}{10}} \operatorname{SNR}_{\mathrm{TH}} N_{0} B$, for $q \in \mathcal{W}$. Therefore, to find the chance-constrained version of (8), it is sufficient to replace $\frac{\mathrm{SNR}_{\mathrm{TH}} N_{0} B}{G\left(q_{i}\right)}$ with $P_{\mathrm{TX}, \min }\left(q_{i}, \chi\right)$. Note that the case of known channel power becomes a special case of (10) for $\sigma\left(q_{i}\right)=0$ (see Appendix A).

Based on the chance-constrained version of (8), we then propose the following optimization problem to find the optimal feasible dynamic coverage policy in the communicationintensive case:

$$
\min _{\mathcal{P}} \sum_{k=1}^{n} \varrho_{k} \mathcal{E}_{k}
$$

s.t.

1) $\alpha_{i, k} t_{i, k}-\rho_{i}\left(\sum_{i \in \mathcal{V}_{k}} t_{i, k}+\frac{\mathrm{d}\left(\mathcal{H}_{k}\right)}{v_{k}}\right) \geq \Delta_{k}, \quad \forall i \in \mathcal{V}_{k}, k=1, \cdots, n$,

2) $\sum_{i \in \mathcal{V}_{k}} t_{i, k}+\frac{\mathrm{d}\left(\mathcal{H}_{k}\right)}{v_{k}} \leq T_{\max }, \quad k=1, \cdots, n$,

3) $P_{\ell, s, k} \sum_{i \in \mathcal{V}_{k}} t_{i, k}+\left(\frac{P_{\ell, m, k}}{v_{k}}+w_{k}\right) \mathrm{d}\left(\mathcal{H}_{k}\right)+t_{c} \sum_{i \in \mathcal{V}_{k}} P_{\mathrm{TX}, i, k} \leq \mathcal{E}_{k}, \quad k=1, \cdots, n$,

4) $P_{\mathrm{TX}, \max , k} \geq P_{\mathrm{TX}, i, k} \geq P_{\mathrm{TX}, \min }\left(q_{i}, \chi\right), \quad \forall i \in \mathcal{V}_{k}, k=1, \cdots, n$,

5) $0 \leq v_{k} \leq v_{\max , k}, \quad k=1, \cdots, n$,

6) $\mathcal{E}_{k} \leq \mathcal{E}_{\max , k}, \quad k=1, \cdots, n$, 
where $\varrho_{k}>0$, for $k=1, \cdots, n$, denote the weights assigned to the agents. The solution of (11) minimizes a weighted sum of the total energy consumptions of the mobile agents in each period, while satisfying the chance-constrained version of (8). Note that feasibility of (11) may not exactly translate to feasibility of the actual dynamic coverage problem, as defined by (8), unless the quality of channel estimation is high and $\chi$ is selected large. Still, we refer to the solution of (11) as the optimal feasible dynamic coverage policy since it provides a good assessment of the feasibility and is the best you can do without the full knowledge of the channel. Also, depending on the channel qualities at the POIs and the thresholds $T_{\max }, P_{\mathrm{TX}, \max , k}$ and $\mathcal{E}_{\max , k}$, a feasible dynamic coverage policy may or may not exist in the communication-intensive case. Finally, choosing positive stability margins $\left(\Delta_{k}>0\right.$, for $\left.k=1, \cdots, n\right)$ increases the robustness of the optimal policy to the effects of unmodeled system parameters. Robustness, however, comes at the cost of consuming more energy, as expected.

\subsection{Optimal Solution of Dynamic Coverage Problem in the Communication-Intensive Case}

In the main theorem of this section (Theorem 3.1), we provide a closed-form expression for the solution of (11), given a partition $\left\{\mathcal{V}_{k}\right\}_{k=1}^{n}$.

THEOREM 3.1. Consider a partition $\left\{\mathcal{V}_{k}\right\}_{k=1}^{n}$ of $\mathcal{V}$. Then, the following are true for the solution of optimization problem (11):

(1) For a given set of non-negative stability margins $\Delta_{k}, k=1, \cdots, n,(11)$ is feasible if and only if the following are true, for $k=1, \cdots, n$ :

1) $P_{\mathrm{TX}, \min }\left(q_{i}, \chi\right) \leq P_{\mathrm{TX}, \max , k}, \quad \forall i \in \mathcal{V}_{k}$,

2) $\sum_{i \in \mathcal{V}_{k}} \frac{\rho_{i}}{\alpha_{i, k}}<1$,

3) $\frac{\mathrm{d}\left(\mathcal{H}_{k}^{*}\right)}{v_{\max , k}} \leq \min \left\{T_{\max } \phi_{k}-\Delta_{k} \eta_{k}, \frac{\left(\overline{\mathcal{E}}_{\max , k}-w_{k} \mathrm{~d}\left(\mathcal{H}_{k}^{*}\right)\right) \phi_{k}-\Delta_{k} \eta_{k} P_{\ell, s, k}}{\left(1-\phi_{k}\right) P_{\ell, s, k}+\phi_{k} P_{\ell, m, k}}\right\}$,

where $\mathcal{H}_{k}^{*}$ denotes the minimum-length Hamiltonian cycle on $\mathcal{V}_{k}, \overline{\mathcal{E}}_{\max , k} \triangleq \mathcal{E}_{\max , k}-$ $t_{c} \sum_{i \in \mathcal{V}_{k}} P_{\mathrm{TX}, \min }\left(q_{i}, \chi\right), \phi_{k} \triangleq 1-\sum_{i \in \mathcal{V}_{k}} \frac{\rho_{i}}{\alpha_{i, k}}$ and $\eta_{k} \triangleq \sum_{i \in \mathcal{V}_{k}} \frac{1}{\alpha_{i, k}}$.

(2) The maximum stability margin that can be selected for each agent $k$, for $k=1, \cdots, n$, is given as follows when $\sum_{i \in \mathcal{V}_{k}} \frac{\rho_{i}}{\alpha_{i, k}}<1$ :

$$
\Delta_{\max , k}=\min \left\{\frac{\overline{\mathcal{E}}_{\max , k}-w_{k} \mathrm{~d}\left(\mathcal{H}_{k}^{*}\right)+\left(P_{\ell, s, k}-P_{\ell, m, k}\right) \frac{\mathrm{d}\left(\mathcal{H}_{k}^{*}\right)}{v_{\max , k}}}{P_{\ell, s, k}}, T_{\max }\right\} \frac{\phi_{k}}{\eta_{k}}-\frac{1}{\eta_{k}} \frac{\mathrm{d}\left(\mathcal{H}_{k}^{*}\right)}{v_{\max , k}} .
$$

(3) If (11) is feasible, the optimal Hamiltonian cycle of the kth agent is the minimum-length Hamiltonian cycle $\mathcal{H}_{k}^{*}$ and its optimal velocity is the maximum velocity $v_{\max , k}$. We also have the following for the rest of the optimal variables:

$$
\begin{aligned}
& P_{\mathrm{TX}, i, k}^{*}=P_{\mathrm{TX}, \min }\left(q_{i}, \chi\right), \quad \forall i \in \mathcal{V}_{k}, \\
& t_{i, k}^{*}=\Delta_{k}\left(\frac{1}{\alpha_{i, k}}+\frac{\rho_{i} \eta_{k}}{\alpha_{i, k} \phi_{k}}\right)+\frac{\rho_{i}}{\alpha_{i, k} \phi_{k}} \frac{\mathrm{d}\left(\mathcal{H}_{k}^{*}\right)}{v_{\max , k}}, \quad \forall i \in \mathcal{V}_{k} \\
& \mathcal{E}_{k}^{*}=P_{\ell, s, k}\left(\frac{\Delta_{k} \eta_{k}}{\phi_{k}}+\frac{1-\phi_{k}}{\phi_{k}} \frac{\mathrm{d}\left(\mathcal{H}_{k}^{*}\right)}{v_{\max , k}}\right)+\left(\frac{P_{\ell, m, k}}{v_{\max , k}}+w_{k}\right) \mathrm{d}\left(\mathcal{H}_{k}^{*}\right)+t_{c} \sum_{i \in \mathcal{V}_{k}} P_{\mathrm{TX}, \min }\left(q_{i}, \chi\right) .
\end{aligned}
$$

Proof. See Appendix B for the proof. 
Theorem 3.1 can be used to determine whether there exists a feasible policy for a given partition $\left\{\mathcal{V}_{k}\right\}_{k=1}^{n}$. Then, we can find the solution of (11) by searching through all the partitions $\left\{\mathcal{V}_{k}\right\}_{k=1}^{n}$ of $\mathcal{V}$ that satisfy (12), and finding the one with minimum $\sum_{k=1}^{n} \varrho_{k} \mathcal{E}_{k}^{*}$. A more efficient alternative for solving (11) is to use a mixed-integer program (MIP) to find the optimal partitions and optimal stopping times for the mobile agents, when maximum allowed velocities and minimum possible transmission powers are used. Generally, there is more than one way to formulate the MIP. Since there are multiple mobile agents, some MIP formulations can be nonlinear, in which case the optimal solution is very challenging to find. Next, we show how to formulate a mixed-integer linear program (MILP), by transforming the nonlinear mixed-integer constraints to linear ones. This makes finding the optimal dynamic coverage policies tractable even for large number of POIs. Note that MILPs can be solved much more efficiently than their nonlinear alternatives.

Let us consider auxiliary binary variables $x_{i, k}$ and $z_{i, j, k}$, for $i, j \in \mathcal{V}$ and $k=1, \cdots, n$. We have $x_{i, k}=1$ whenever the $i$ th POI is assigned to the $k$ th mobile agent, and $x_{i, k}=0$ otherwise. Also, $z_{i, j, k}=1$ if there exists an edge between the $i$ th and $j$ th POIs in the Hamiltonian cycle assigned to the $k$ th mobile agent, and $z_{i, j, k}=0$ otherwise. To guarantee that every POI that is on the Hamiltonian cycle $\mathcal{V}_{k}$ have one degree in and one degree out, we can add the following set of constraints: $\sum_{j=1, j \neq i}^{m} z_{i, j, k}=x_{i, k}, \sum_{j=1, j \neq i}^{m} z_{j, i, k}=x_{i, k}$, for all $i \in \mathcal{V}$. Then, by defining $d_{i, j} \triangleq\left\|q_{i}-q_{j}\right\|$, we have the following constraints for the period and total energy per period of the $k$ th agent:

$$
\begin{aligned}
& \sum_{i=1}^{m} x_{i, k} t_{i, k}+\frac{1}{v_{\max , k}} \sum_{i=1}^{m} \sum_{j=1, j \neq i}^{m} z_{i, j, k} d_{i, j} \leq T_{\max }, \\
& P_{\ell, s, k} \sum_{i=1}^{m} x_{i, k} t_{i, k}+\left(\frac{P_{\ell, m, k}}{v_{\max , k}}+w_{k}\right) \sum_{i=1}^{m} \sum_{j=1, j \neq i}^{m} z_{i, j, k} d_{i, j}+t_{c} \sum_{i=1}^{m} x_{i, k} P_{\mathrm{TX}, \min }\left(q_{i}, \chi\right) \leq \mathcal{E}_{\max , k},
\end{aligned}
$$

with $t_{i, k} \geq 0$ for all $i, k$. As can be seen, the left-hand side of both constraints are nonlinear functions of $t_{i, k}$ and $x_{i, k}$. In order to make the constraints linear, we consider a large constant $\Omega>0$. We then add the linear constraints $t_{i, k} \leq x_{i, k} \Omega$, for all $i, k$, to guarantee that $t_{i, k}=0$ whenever $x_{i, k}=0\left(i \notin \mathcal{V}_{k}\right)$, while there is no constraint on $t_{i, k}$ whenever $x_{i, k}=1\left(i \in \mathcal{V}_{k}\right)$. This way we can replace the nonlinear term $x_{i, k} t_{i, k}$ with $t_{i, k}$ in (15), without changing the optimal solution. Furthermore, since the $k$ th mobile agent does not need to stabilize any POI out of $\mathcal{V}_{k}$, we should modify the stability constraints of the $k$ th mobile agent such that they automatically become true for all $i \notin \mathcal{V}_{k}$. This is done by considering the following stability constraints:

$$
\alpha_{i, k} t_{i, k}-\rho_{i}\left(\sum_{i=1}^{m} t_{i, k}+\frac{1}{v_{\max , k}} \sum_{i=1}^{m} \sum_{j=1, j \neq i}^{m} z_{i, j, k} d_{i, j}\right)+\left(1-x_{i, k}\right) \Omega \geq \Delta_{k}, \quad \forall i, k
$$

We can see that when $x_{i, k}=0$, constraint (16) becomes true if $\Omega>0$ is large enough. It is easy to confirm that any $\Omega \geq \max \left\{T_{\max },\left(\max _{i} \rho_{i}\right) T_{\max }+\max _{k} \Delta_{k}\right\}$ can be considered large enough for this set of constraints. Such an $\Omega$ also guarantees that whenever $x_{i, k}=1$, the constraint $t_{i, k} \leq x_{i, k} \Omega$ is always true. Based on this discussion, the MILP formulation for solving (11) is given by Program 1.

Constraints 1, 2 and 3 in Program 1 are the stability, time and energy constraints, as introduced before. Constraint 4 forces each POI $i \in \mathcal{V}$ to have exactly one degree in and 
PROGRAM 1: MILP for finding optimal dynamic coverage policy in the communicationintensive case $\min \sum_{k=1}^{n} \varrho_{k} \mathcal{E}_{k}$, s.t.

1) $\alpha_{i, k} t_{i, k}-\rho_{i}\left(\sum_{i=1}^{m} t_{i, k}+\frac{1}{v_{\max , k}} \sum_{i=1}^{m} \sum_{j=1, j \neq i}^{m} z_{i, j, k} d_{i, j}\right)+\left(1-x_{i, k}\right) \Omega \geq \Delta_{k}, \forall i, k$,

2) $\sum_{i=1}^{m} t_{i, k}+\frac{1}{v_{\max , k}} \sum_{i=1}^{m} \sum_{j=1, j \neq i}^{m} z_{i, j, k} d_{i, j} \leq T_{\max }, \quad \forall k$

3) $P_{\ell, s, k} \sum_{i=1}^{m} t_{i, k}+\left(\frac{P_{\ell, m, k}}{v_{\max , k}}+w_{k}\right) \sum_{i=1}^{m} \sum_{j=1, j \neq i}^{m} z_{i, j, k} d_{i, j}+t_{c} \sum_{i=1}^{m} x_{i, k} P_{\mathrm{TX}, \min }\left(q_{i}, \chi\right) \leq \mathcal{E}_{k}, \forall k$,

4) $\sum_{j=1, j \neq i}^{m} z_{i, j, k}=x_{i, k}, \sum_{j=1, j \neq i}^{m} z_{j, i, k}=x_{i, k}, \quad \forall i, k$,

5) $\sum_{k=1}^{n} x_{i, k}=1, \quad \forall i$,

6) $\sum_{i=1}^{m} e_{i}=n$,

7) $u_{i}-u_{j}-m\left(e_{i}+e_{j}\right)+(m-1) \sum_{k=1}^{n} z_{i, j, k} \leq m-2, \quad \forall i, j \neq i$,

8) $2-e_{i} \leq u_{i} \leq m, \quad \forall i$,

9) $t_{i, k} \leq \bar{x}_{i, k} \Omega, \quad \forall i, k$,

10) $x_{i, k} P_{\mathrm{TX}, \min }\left(q_{i}, \chi\right) \leq P_{\mathrm{TX}, \max , k}, \quad \forall i, k$,

11) $\mathcal{E}_{k} \leq \mathcal{E}_{\max , k}, \quad \forall k$,

12) $z_{i, j, k} \in\{0,1\}, x_{i, k} \in\{0,1\}, e_{i} \in\{0,1\}, u_{i} \in \mathbb{N}, 0 \leq t_{i, k} \leq \Omega, \quad \forall i, j, k$,

one degree out. Constraint 5 guarantees that each POI is assigned to one mobile agent. ${ }^{7}$ Constraints 6, 7 and 8 are the sub-tour elimination constraints (SECs), which are added to prevent any invalid sub-tour on the set of POIs assigned to each agent [Gutin and Punnen 2004]. To prevent sub-tours, we have introduced $2 m$ auxiliary variables $e_{i}$ and $u_{i}$, for $i \in \mathcal{V}$, and used a modified version of the well-known Miller-Tucker-Zemlin (MTZ) constraints [Gutin and Punnen 2004; Na 2007]. Constraint 9 forces $t_{i, k}=0$ whenever $x_{i, k}=0$. Constraint 10 is the transmission power constraint, which implies that if a POI is assigned to a mobile agent, that mobile agent should be connected with a probability larger than $\chi$ at the POI. Finally, constraint 11 is the constraint on the maximum total energy consumption in each period. Note that the number of the POIs is assumed larger than the number of the mobile agents $(m>n)$.

Solving the MILP of Program 1 is NP-hard and, therefore, the computational complexity of finding the optimal solution increases exponentially as a function of the number of POIs and/or the number of mobile agents. However, the fact that the proposed dynamic coverage problem becomes an MILP is helpful as there exist many solvers (such as IBM ILOG CPLEX [CPL ] and SAS/OR [SAS ]) that can solve large scale MILPs very efficiently.

It is worth mentioning that MTZ formulations for sub-tour elimination, as used in vehicle routing problem (VRP) or multiple traveling salesman problem (mTSP), typically assume a fixed POI, called depot, through which all the mobile agents must pass [Bektas 2006]. The MTZ formulation used in Program 1 is different from those formulations, as it assumes no depot [Na 2007]. The idea here is to introduce floating depot variables $e_{i}$, for $i \in \mathcal{V}$, which guarantee that whenever $e_{i}=1$ (the $i$ th POI is selected as a depot), constraint 7 in Program 1 is always true. Also note that the MTZ formulation has a polynomial size (i.e., the number of SECs is of polynomial order), compared to the exponential size of several alternative formulations in the literature [Bektas 2006].

Next, we continue with solving the dynamic coverage problem in the communicationefficient case.

\footnotetext{
${ }^{7}$ Note that if $x_{i, k}=1$, the $k$ th mobile agent needs to visit at least one more POI (other than POI $i$ ) to satisfy constrain 4 in Program 1. Therefore, the case of one single POI assigned to one agent is automatically prevented, i.e., $\left|\mathcal{V}_{k}^{*}\right|>1$ for all $k$.
} 


\section{DYNAMIC COVERAGE OF TIME-VARYING ENVIRONMENTS IN THE COMMUNICATION-EFFICIENT CASE}

Unlike the communication-intensive case, the mobile agents in the communication-efficient case are required to be connected only once along their trajectories. In this case, the communication points $\xi_{\mathrm{TX}, k}$, for $k=1, \cdots, n$, are extra design variables that need to be optimized along with the ones already introduced in the communication-intensive case. A dynamic coverage policy in the communication-efficient case is defined as follows:

Definition 4.1. A dynamic coverage policy for the $k$ th mobile agent in the communication-efficient case is a tuple $\mathcal{P}_{k}=\left(\mathcal{V}_{k}, \mathcal{H}_{k}, v_{k}, \xi_{\mathrm{TX}, k}, P_{\mathrm{TX}, k},\left\{t_{\mathrm{TX}, i, k}\right\}_{i \in \mathcal{V}_{k}}\right)$. The overall dynamic coverage policy to find is then the tuple $\mathcal{P}=\left(\mathcal{P}_{1}, \cdots, \mathcal{P}_{n}\right)$.

Similarly, a dynamic coverage policy $\mathcal{P}$ is feasible in the communication-efficient case if the following are true:

$$
\begin{aligned}
& \text { 1) } \alpha_{i, k} t_{i, k}-\rho_{i}\left(\sum_{i \in \mathcal{V}_{k}} t_{i, k}+\frac{\mathrm{d}\left(\mathcal{H}_{k}\right)}{v_{k}}\right) \geq \Delta_{k}, \quad \forall i \in \mathcal{V}_{k}, k=1, \cdots, n, \\
& \text { 2) } \sum_{i \in \mathcal{V}_{k}} t_{i, k}+\frac{\mathrm{d}\left(\mathcal{H}_{k}\right)}{v_{k}} \leq T_{\max }, \quad k=1, \cdots, n, \\
& \text { 3) } P_{\ell, s, k} \sum_{i \in \mathcal{V}_{k}} t_{i, k}+\left(\frac{P_{\ell, m, k}}{v_{k}}+w_{k}\right) \mathrm{d}\left(\mathcal{H}_{k}\right)+t_{c} P_{\mathrm{TX}, k} \leq \mathcal{E}_{\max , k}, \quad k=1, \cdots, n, \\
& \text { 4) } P_{\mathrm{TX}, \max , k} \geq P_{\mathrm{TX}, i, k} \geq \frac{\mathrm{SNR}_{\mathrm{TH}} N_{0} B}{G\left(\xi_{\mathrm{TX}, k}\right)}, \quad k=1, \cdots, n, \\
& \text { 5) } 0 \leq v_{k} \leq v_{\max , k}, \quad k=1, \cdots, n .
\end{aligned}
$$

Note that based on Lemma 3.1, the dynamic coverage policy can be stabilized if each mobile agent $k$ is connected at the communication point $\xi_{\mathrm{TX}, k}$. Following the stochastic programming approach of Section 3, the optimal feasible dynamic coverage policy in the communication-efficient case is given by the solution of the following optimization problem:

$$
\min _{\mathcal{P}} \sum_{k=1}^{n} \varrho_{k} \mathcal{E}_{k}
$$

s.t.

$$
\begin{aligned}
& \text { 1) } \alpha_{i, k} t_{i, k}-\rho_{i}\left(\sum_{i \in \mathcal{V}_{k}} t_{i, k}+\frac{\mathrm{d}\left(\mathcal{H}_{k}\right)}{v_{k}}\right) \geq \Delta_{k}, \quad \forall i \in \mathcal{V}_{k}, k=1, \cdots, n, \\
& \text { 2) } \sum_{i \in \mathcal{V}_{k}} t_{i, k}+\frac{\mathrm{d}\left(\mathcal{H}_{k}\right)}{v_{k}} \leq T_{\max }, \quad k=1, \cdots, n, \\
& \text { 3) } P_{\ell, s, k} \sum_{i \in \mathcal{V}_{k}} t_{i, k}+\left(\frac{P_{\ell, m, k}}{v_{k}}+w_{k}\right) \mathrm{d}\left(\mathcal{H}_{k}\right)+t_{c} P_{\mathrm{TX}, k} \leq \mathcal{E}_{k}, \quad k=1, \cdots, n, \\
& \text { 4) } P_{\mathrm{TX}, \max , k} \geq P_{\mathrm{TX}, i, k} \geq P_{\mathrm{TX}, \min }\left(\xi_{\mathrm{TX}, k}, \chi\right), \quad k=1, \cdots, n, \\
& \text { 5) } 0 \leq v_{k} \leq v_{\max , k}, \quad k=1, \cdots, n, \\
& \text { 6) } \mathcal{E}_{k} \leq \mathcal{E}_{\max , k}, \quad k=1, \cdots, n,
\end{aligned}
$$

where $P_{\mathrm{TX}, \min }(q, \chi)$, for any $q \in \mathcal{W}$, is defined in (10). The solution of (18) minimizes a weighted sum of the total energy consumptions of the mobile agents in each period, while satisfying the chanced-constrained version of (17). Similar to the communication- 
intensive case, we emphasize that a feasible dynamic coverage policy may not exist in the communication-efficient case, depending on the channel qualities at the POIs and the thresholds $T_{\max }, P_{\mathrm{TX}, \max , k}$ and $\mathcal{E}_{\max , k}$. Next we show how to find the optimal solution of (18) using an MILP, in case the communication point for each agent is selected to be at one of the POIs assigned to it.

\subsection{Optimal Solution of Dynamic Coverage Problem in the Communication-Efficient Case}

Generally, the communication point $\xi_{\mathrm{TX}, k}$ can be any point on the trajectory of the $k$ th agent. Finding the optimal dynamic coverage policy in this general case is, however, very challenging. This is due to the fact that given the partition $\left\{\mathcal{V}_{k}\right\}_{k=1}^{n}$ and conditioned on the channel power over the workspace, the optimal Hamiltonian cycle for an agent $k$ may become different from the minimum-length Hamiltonian cycle $\mathcal{H}_{k}^{*}$. In other words, since there is no requirement for transmission at the POIs, moving to a point out of the minimum-length Hamiltonian cycle can possibly minimize the communication energy and the resulting overall energy consumption. In order to simplify the problem, we consider the following assumption in this section:

Assumption 4.1. The communication point $\xi_{\mathrm{TX}, k}$ is selected to be at one of the POIs in $\mathcal{V}_{k}$, i.e., $\xi_{\mathrm{TX}, k}=q_{i}$, for some $i \in \mathcal{V}_{k}$.

Based on this assumption and given a partition $\left\{\mathcal{V}_{k}\right\}_{k=1}^{n}$, the optimal policy in the communication-efficient case is then given by the following theorem:

TheOREM 4.1. Consider a partition $\left\{\mathcal{V}_{k}\right\}_{k=1}^{n}$ of $\mathcal{V}$. Then, the following are true for the solution of optimization problem (18):

(1) For a given set of non-negative stability margins $\Delta_{k}, k=1, \cdots, n$, (18) is feasible if and only if the following are true, for $k=1, \cdots, n$ :

1) $\min _{i \in \mathcal{V}_{k}} P_{\mathrm{TX}, \min }\left(q_{i}, \chi\right) \leq P_{\mathrm{TX}, \max , k}$,

2) $\sum_{i \in \mathcal{V}_{k}} \frac{\rho_{i}}{\alpha_{i, k}}<1$,

3) $\frac{\mathrm{d}\left(\mathcal{H}_{k}^{*}\right)}{v_{\max , k}} \leq \min \left\{T_{\max } \phi_{k}-\Delta_{k} \eta_{k}, \frac{\left(\overline{\mathcal{E}}_{\max , k}-w_{k} \mathrm{~d}\left(\mathcal{H}_{k}^{*}\right)\right) \phi_{k}-\Delta_{k} \eta_{k} P_{\ell, s, k}}{\left(1-\phi_{k}\right) P_{\ell, s, k}+\phi_{k} P_{\ell, m, k}}\right\}$,

where $\mathcal{H}_{k}^{*}$ denotes the minimum-length Hamiltonian cycle on $\mathcal{V}_{k}, \overline{\mathcal{E}}_{\max , k} \triangleq \mathcal{E}_{\max , k}-$ $t_{c} \min _{i \in \mathcal{V}_{k}} P_{\mathrm{TX}, \min }\left(q_{i}, \chi\right), \phi_{k} \triangleq 1-\sum_{i \in \mathcal{V}_{k}} \frac{\rho_{i}}{\alpha_{i, k}}$ and $\eta_{k} \triangleq \sum_{i \in \mathcal{V}_{k}} \frac{1}{\alpha_{i, k}}$.

(2) The maximum stability margin that can be selected for each agent $k$, for $k=1, \cdots, n$, is given as follows when $\sum_{i \in \mathcal{V}_{k}} \frac{\rho_{i}}{\alpha_{i, k}}<1$ :

$$
\Delta_{\max , k}=\min \left\{\frac{\overline{\mathcal{E}}_{\max , k}-w_{k} \mathrm{~d}\left(\mathcal{H}_{k}^{*}\right)+\left(P_{\ell, s, k}-P_{\ell, m, k}\right) \frac{\mathrm{d}\left(\mathcal{H}_{k}^{*}\right)}{v_{\max , k}}}{P_{\ell, s, k}}, T_{\max }\right\} \frac{\phi_{k}}{\eta_{k}}-\frac{1}{\eta_{k}} \frac{\mathrm{d}\left(\mathcal{H}_{k}^{*}\right)}{v_{\max , k}} .
$$

(3) If (18) is feasible, the optimal Hamiltonian cycle of the kth agent is the minimum-length Hamiltonian cycle $\mathcal{H}_{k}^{*}$ and its optimal velocity is the maximum velocity $v_{\max , k}$. We also 
have the following for the rest of the optimal variables:

$$
\begin{aligned}
& P_{\mathrm{TX}, k}^{*}=\min _{i \in \mathcal{V}_{k}} P_{\mathrm{TX}, \min }\left(q_{i}, \chi\right) \text { and } \xi_{\mathrm{TX}, k}^{*}=q_{i k}^{*}, \text { for } i_{k}^{*}=\operatorname{argmin}_{i \in \mathcal{V}_{k}} P_{\mathrm{TX}, \min }\left(q_{i}, \chi\right), \\
& t_{i, k}^{*}=\Delta_{k}\left(\frac{1}{\alpha_{i, k}}+\frac{\rho_{i} \eta_{k}}{\alpha_{i, k} \phi_{k}}\right)+\frac{\rho_{i}}{\alpha_{i, k} \phi_{k}} \frac{\mathrm{d}\left(\mathcal{H}_{k}^{*}\right)}{v_{\max , k}}, \quad \forall i \in \mathcal{V}_{k}, \\
& \mathcal{E}_{k}^{*}=P_{\ell, s, k}\left(\frac{\Delta_{k} \eta_{k}}{\phi_{k}}+\frac{1-\phi_{k}}{\phi_{k}} \frac{\mathrm{d}\left(\mathcal{H}_{k}^{*}\right)}{v_{\max , k}}\right)+\left(\frac{P_{\ell, m, k}}{v_{\max , k}}+w_{k}\right) \mathrm{d}\left(\mathcal{H}_{k}^{*}\right)+t_{c} \min _{i \in \mathcal{V}_{k}} P_{\mathrm{TX}, \min }\left(q_{i}, \chi\right) .
\end{aligned}
$$

Proof. The proof is similar to the proof of Theorem 3.1 and is omitted for brevity.

Program 2 then shows an MILP formulation for solving (18). This MILP can be used to find the optimal partitions, optimal cycles, optimal communication points and optimal stopping times of the mobile agents, when the optimal velocities and transmission powers are used. Note that, as compared to the communication-intensive case, the MILP formulation is more complicated in this case, since the optimal transmission points of the mobile agent are not known beforehand.

PROGRAM 2: MILP for finding optimal dynamic coverage policy in the communication-efficient case

$\min \sum_{k=1}^{n} \varrho_{k} \mathcal{E}_{k}$,

s.t.

1) $\alpha_{i, k} t_{i, k}-\rho_{i}\left(\sum_{i=1}^{m} t_{i, k}+\frac{1}{v_{\max , k}} \sum_{i=1}^{m} \sum_{j=1, j \neq i}^{m} z_{i, j, k} d_{i, j}\right)+\left(1-x_{i, k}\right) \Omega^{\prime} \geq \Delta_{k}, \forall i, k$,

2) $\sum_{i=1}^{m} t_{i, k}+\frac{1}{v_{\max , k}} \sum_{i=1}^{m} \sum_{j=1, j \neq i}^{m} z_{i, j, k} d_{i, j} \leq T_{\max }, \quad \forall k$,

3) $P_{\ell, s, k} \sum_{i=1}^{m} t_{i, k}+\left(\frac{P_{\ell, m, k}}{v_{\max , k}}+w_{k}\right) \sum_{i=1}^{m} \sum_{j=1, j \neq i}^{m} z_{i, j, k} d_{i, j}+t_{c} \sum_{i=1}^{m} y_{i, k} P_{\mathrm{TX}, \min }\left(q_{i}, \chi\right) \leq \mathcal{E}_{k}, \forall k$,

4) $\sum_{j=1, j \neq i}^{m} z_{i, j, k}=x_{i, k}, \sum_{j=1, j \neq i}^{m} z_{j, i, k}=x_{i, k}, \quad \forall i, k$,

5) $\sum_{k=1}^{n} x_{i, k}=1, \quad \forall i$

6) $\sum_{i=1}^{m} e_{i}=n$,

7) $u_{i}-u_{j}-m\left(e_{i}+e_{j}\right)+(m-1) \sum_{k=1}^{n} z_{i, j, k} \leq m-2, \quad \forall i, j \neq i$,

8) $2-e_{i} \leq u_{i} \leq m, \quad \forall i$,

9) $t_{i, k} \leq x_{i, k} \Omega^{\prime}, \quad \forall i, k$,

10) $\sum_{i=1}^{m} y_{i, k}=1, \quad \forall k$,

11) $y_{i, k} \leq x_{i, k} \Omega^{\prime}, \quad \forall i, k$,

12) $y_{i, k} P_{\mathrm{TX}, \min }\left(q_{i}, \chi\right) \leq P_{\mathrm{TX}, \max , k}, \quad \forall i, k$,

13) $\mathcal{E}_{k} \leq \mathcal{E}_{\max , k}, \quad \forall k$

14) $z_{i, j, k} \in\{0,1\}, x_{i, k} \in\{0,1\}, y_{i, k} \in\{0,1\}, e_{i} \in\{0,1\}, u_{i} \in \mathbb{N}, 0 \leq t_{i, k} \leq \Omega^{\prime}, \forall i, j, k$,

In Program 2, the constant $\Omega^{\prime}$ is selected large enough, similar to $\Omega$ in Program 1. Furthermore, in addition to the variables used in Program 1, we have introduced $m n$ auxiliary binary variables $y_{i, k}$, for $i \in \mathcal{V}$ and $k=1, \cdots, n$. For each mobile agent $k, y_{i, k}=1$ if the $i$ th POI is selected as the communication point, and $y_{i, k}=0$ otherwise. Constraint 10 in Program 2 guarantees that only one POI in $\mathcal{V}_{k}$ is selected as the communication point. Constraint 11 also forces $y_{i, k}=0$ whenever $x_{i, k}=0$. Explanation of other constraints is similar to Program 1. Also, similar to the communication-intensive case, the number of the POIs is assumed larger than the number of the mobile agents $(m>n)$. 


\subsection{Virtual POls in the Communication-Efficient Case}

The coverage task in the communication-efficient case can be feasibly stabilized if at least one POI is connected along the Hamiltonian cycle of each mobile agent (as opposed to all POIs in the communication-intensive case). In case there is no feasible dynamic coverage policy in the communication-efficient case, due to a poor channel quality at the POIs, we may be able to feasibly stabilize the coverage task by adding a number of virtual POIs. These are points close enough to the actual POIs, which have a good channel quality. Adding virtual POIs does not guarantee the existence of a feasible coverage policy. It, however, increases the chance of finding such a policy in case the channel quality is low at the positions of the actual POIs.

\section{PROBABILISTIC ANALYSIS OF THE DYNAMIC COVERAGE PROBLEM}

In this section, we complete our dynamic coverage framework by probabilistically analyzing some of the properties of the considered dynamic coverage problem, such as average minimum energy required for coverage or maximum number of POIs that can be covered by a mobile agent. In other words, we want to answer the following questions: if the positions of the POIs assigned to an agent and the channel powers at the POIs are distributed according to certain distributions, what is the maximum number of POIs that can be covered by that agent and what is the average minimum total energy consumed by the agent to cover a given number of POIs? Such analysis can help considerably in the planning phase by providing a priori knowledge about the dynamic coverage performance before the deployment of the mobile agents. Note that probabilistic analysis is very common in both wireless communications and TSP literature. For instance, average performance metrics are characterized for a case that a cell phone user is going to operate in an environment where the channel has a certain distribution (see Chapter 6 of [Goldsmith 2005]). Also, probabilistic analysis of TSP is performed for a given spatial distribution for the POIs [Bullo et al. 2011; Gutin and Punnen 2004]. The contribution of this section is then to provide a probabilistic performance analysis for our networked dynamic coverage problem considering the distribution of the communication links and the spatial distribution of POIs.

Next, we build on our communication-intensive and communication-efficient results and derive conditions for a dynamic coverage policy to be feasible with a large probability, in case the channel powers at the POIs as well as the positions of the POIs assigned to the agent are stochastic and drawn from certain distributions. We then find the maximum number of POIs that can be assigned to the agent given limited energy and time budgets, as well as the average minimum energy required to feasibly cover a given number of POIs. Without loss of generality, in this section we assume that $\rho_{i}$ and $\alpha_{i, k}$ are constant, i.e., $\rho_{i}=\rho$ and $\alpha_{i, k}=\alpha_{k}$, for $i \in \mathcal{V}$ and $k=1, \cdots, n$. We furthermore assume that $P_{\ell, s, k}=P_{\ell, m, k}=P_{\ell, k}$, for $k=1, \cdots, n{ }^{8}$ Also, in order to better follow the discussion, we assume that the channel is known, i.e., for any realization of the channel and for any given set of POIs, the agents plan based on the full knowledge of the channel.

Note that, given the distributions of the channel powers and the positions of the POIs assigned to each agent, our main goal is to find the probability of having a feasible dynamic coverage policy and derive conditions that guarantee this probability is larger than a threshold. More specifically, we are interested in satisfying the following:

$$
\mathbb{P}\{\text { Constraints } 1 \text { to } 5 \text { of }(8) \text { or }(17) \text { hold }\} \geq \chi
$$

\footnotetext{
${ }^{8}$ Note that these assumptions are made to simplify the theoretical analysis of this section. Similar results can be found for the case that either one of these assumptions does not hold. For instance, the results of this section can be easily extended to the case that $\rho_{i}$, for $i=1, \cdots, n$, are i.i.d. random variables, independent of the channel and the positions of the POIs, or the case where $P_{\ell, s, k} \neq P_{\ell, m, k}$.
} 
Deriving such conditions for our networked dynamic coverage problem without any simplification is, however, considerably challenging. Therefore, similar to Section 3 and Section 4, we use a sub-optimal approach based on stochastic programming, i.e., we replace any stochastic constraint in (8) or (17) with its chance constraint, while treating the rest of the variables, aside from channel powers and the positions of the POIs, deterministically.

Consider the $k$ th mobile agent to which $m_{k}$ POIs are assigned. Based on the wellestablished probabilistic channel models, we assume that the pdf of the channel power (in the $\mathrm{dB}$ domain) at any position $q \in \mathcal{W}$ is given by a Gaussian distribution with mean $\widetilde{G}_{\mathrm{dB}}(q)$ and variance $\widetilde{\sigma}^{2}(q) .{ }^{9}$ Moreover, we assume that the joint pdf of the channel powers at any given set of positions $Q_{k} \triangleq\left[q_{1}^{\mathrm{T}}, \cdots, q_{m_{k}}^{\mathrm{T}}\right]^{\mathrm{T}}$, corresponding to the positions of the POIs assigned to the $k$ th agent, is given by a multi-variate Gaussian distribution with $m_{k} \times 1$ mean vector $\widetilde{G}_{\mathrm{dB}}\left(Q_{k}\right)$ and $m_{k} \times m_{k}$ covariance matrix $\widetilde{\Sigma}\left(Q_{k}\right)$. As for the pdf of the positions of the POIs assigned to the $k$ th agent, we consider a large $e^{10}$ number of POIs that are independently and identically distributed (i.i.d.) according to an absolutely continuous pdf $\psi(q)$. The following result from the probabilistic traveling salesman problem (PTSP) literature is also used extensively in the rest of this section:

Lemma 5.1. Consider a single mobile agent, e.g., the kth one. Assume that the POIs assigned to this agent are i.i.d. according to an absolutely continuous pdf $\psi(q)$ defined over the compact set $\mathcal{W}$. Then, there exists a constant $\theta_{\mathrm{TSP}}$ such that

$$
\begin{aligned}
& \mathbb{P}\left\{\lim _{m_{k} \rightarrow \infty} \frac{\mathrm{d}\left(\mathcal{H}_{k}^{*}\right)}{\sqrt{m_{k}}}=\theta_{\mathrm{TSP}} \int_{\mathcal{W}} \sqrt{\psi(q)} d q\right\}=1, \\
& \lim _{m_{k} \rightarrow \infty} \frac{\mathbb{E}\left\{\mathrm{d}\left(\mathcal{H}_{k}^{*}\right)\right\}}{\sqrt{m_{k}}}=\theta_{\mathrm{TSP}} \int_{\mathcal{W}} \sqrt{\psi(q)} d q,
\end{aligned}
$$

where $m_{k}=\left|\mathcal{V}_{k}\right|$ is the number of POIs assigned to the kth mobile agent.

Proof. See [Gutin and Punnen 2004].

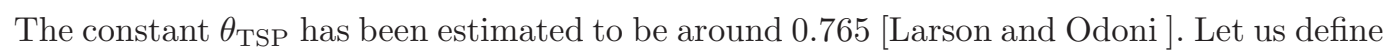
$\zeta \triangleq \theta_{\operatorname{TSP}} \int_{\mathcal{W}} \sqrt{\psi(q)} d q$. Lemma 5.1 implies that asymptotically $\mathrm{d}\left(\mathcal{H}_{k}^{*}\right)$ converges to $\zeta \sqrt{m_{k}}$ with probability one. For convex environments, $\zeta \sqrt{m_{k}}$ is a tight approximation for $\mathrm{d}\left(\mathcal{H}_{k}^{*}\right)$ for $m_{k}$ as small as 15 [Larson and Odoni ]. The assumption of large number of POIs in this section is then equivalent to $m_{k} \geq 15$, for $k=1, \cdots, n$, which is required to guarantee that $\zeta \sqrt{m_{k}}$ remains a tight approximation for $\mathrm{d}\left(\mathcal{H}_{k}^{*}\right)$. Next, we continue with probabilistic analysis of the dynamic coverage problem in the communication-intensive case.

\subsection{Probabilistic Analysis of the Dynamic Coverage Problem in the Communication-Intensive Case}

Let us start with finding the probability of connectivity of the $k$ th mobile agent at the $i$ th POI in the communication-intensive case, when $q_{i}$ is a random variable and distributed according to pdf $\psi(q)$. We calculate this by first conditioning on $q_{i}$ and then averaging over its distribution:

$$
\mathbb{P}\left\{\frac{P_{\mathrm{TX}, i, k} G\left(q_{i}\right)}{N_{0} B} \geq \mathrm{SNR}_{\mathrm{TH}}\right\}=\int_{\mathcal{W}} Q\left(\frac{10 \log _{10}\left(\frac{\mathrm{SNR}_{\mathrm{TH}} N_{0} B}{P_{\mathrm{TX}, i, k}}\right)-\widetilde{G}_{\mathrm{dB}}\left(q_{i}\right)}{\widetilde{\sigma}\left(q_{i}\right)}\right) \psi(q) d q .
$$

\footnotetext{
${ }^{9}$ We assume a general Gaussian distribution for the channel power in this section. A special case of such channel distribution is the one given by our probabilistic channel assessment framework, which we used in Section 3 and Section 4 . In that case, $\widetilde{G}_{\mathrm{dB}}(q)=\hat{G}_{\mathrm{dB}}(q)$ and $\widetilde{\sigma}(q)=\sigma(q)$.

${ }^{10}$ The meaning of a large number of POIs will be explained shortly in this section.
} 
By using the fact that the right-hand side of (24) is an increasing function of $P_{\mathrm{TX}, i, k}$, we have the following condition to guarantee $\mathbb{P}\left\{\frac{P_{\mathrm{TX}, i, k} G\left(q_{i}\right)}{N_{0} B} \geq \mathrm{SNR}_{\mathrm{TH}}\right\} \geq \chi$ :

$$
P_{\mathrm{TX}, i, k} \geq 10^{-\breve{G}_{\mathrm{dB}}(\chi) / 10} \mathrm{SNR}_{\mathrm{TH}} N_{0} B \triangleq \widetilde{P}_{\mathrm{TX}, \min }(\chi), \quad i \in \mathcal{V}_{k}, k=1, \cdots, n,
$$

where $\breve{G}_{\mathrm{dB}}(\chi)$ is the unique solution to the following equation as a function of $G$ :

$$
\int_{\mathcal{W}} Q\left(\frac{G-\widetilde{G}_{\mathrm{dB}}(q)}{\widetilde{\sigma}(q)}\right) \psi(q) d q=\chi
$$

Consequently, in the chance-constrained version of (8) in this case, it is sufficient to replace $\frac{\mathrm{SNR}_{\mathrm{TH}} N_{0} B}{G\left(q_{i}\right)}$ in constraint 4 with $\widetilde{P}_{\mathrm{TX}, \min }(\chi)$.

Next consider the constraints 1,2 and 3 in (8). It can be seen that given $m_{k}$, the only stochastic quantity in these constraints is $\mathrm{d}\left(\mathcal{H}_{k}\right)$. From Theorem 3.1, we know that, for a given set of POIs, the optimal Hamiltonian cycle for the $k$ th is the minimum-length Hamiltonian cycle $\mathcal{H}_{k}^{*}$. Without loss of generality, we can therefore replace $\mathrm{d}\left(\mathcal{H}_{k}\right)$ with $\mathrm{d}\left(\mathcal{H}_{k}^{*}\right)$ in these constraints. On the other hand, from Lemma 5.1, $\mathrm{d}\left(\mathcal{H}_{k}^{*}\right)$ converges to $\zeta \sqrt{m_{k}}$. If $m_{k}$ is large enough such that the $\mathrm{d}\left(\mathcal{H}_{k}^{*}\right)$ is approximately equal to $\zeta \sqrt{m_{k}}$, we can then replace $\mathrm{d}\left(\mathcal{H}_{k}\right)$ with $\zeta \sqrt{m_{k}}$ in constraints 1,2 and 3 in (8) to form the chance constraints.

Based on these results, we can conclude that to form the chance-constrained version of (8) in this case, it is sufficient to replace $\mathrm{d}\left(\mathcal{H}_{k}\right)$ with $\zeta \sqrt{m_{k}}$ and $\frac{\operatorname{SNR}_{\mathrm{TH}} N_{0} B}{G\left(q_{i}\right)}$ with $\widetilde{P}_{\mathrm{TX}, \min }(\chi)$, assuming that $m_{k}$ remains large. Furthermore, the results of Theorem 3.1 hold in this case too, provided that the same replacement is done. Given a large $m_{k}$, this implies that there exists at least one feasible solution that satisfies the chance-constrained version of (8) if the following are true, for $k=1, \cdots, n$ :

1) $\widetilde{P}_{\mathrm{TX}, \min }(\chi) \leq P_{\mathrm{TX}, \max , k}$,

2) $m_{k}<\frac{\alpha_{k}}{\rho}$

3) $\min \left\{\frac{\mathcal{E}_{\mathrm{max}, k}-t_{c} m_{k} \widetilde{P}_{\mathrm{TX}, \min }(\chi)-w_{k} \zeta \sqrt{m_{k}}}{P_{\ell, k}}, T_{\max }\right\}\left(1-m_{k} \frac{\rho}{\alpha_{k}}\right) \geq \frac{\zeta \sqrt{m_{k}}}{v_{\max , k}}$.

Note that to find the conditions in $(27)$, we set $\Delta_{k}=0$. By setting $\Delta_{k}=0$, we find the least restrictive feasibility conditions. Also, since $m_{k}$ is assumed large, it is necessary (but not sufficient) to assume a large $\frac{\alpha_{k}}{\rho}$ in order to satisfy (27).

5.1.1. An Upper Bound on the Maximum Number of POls Covered by a Mobile Agent in the Communication-Intensive Case. The conditions in (27) can be used to characterize an upper bound on the maximum number of POIs that can be covered by a mobile agent, which is an important performance metric for a given dynamic coverage task. Consider the following theorem:

THEOREM 5.1. Assume that for the $k$ th agent and for the given $\chi$, we have $\widetilde{P}_{\mathrm{TX}, \min }(\chi) \leq$ $P_{\mathrm{TX}, \max , k}$, where $\widetilde{P}_{\mathrm{TX}, \min }(\chi)$ is given by (25). Then, an upper-bound on the maximum number of POIs that can be assigned to the kth agent in the communication-intensive case, to satisfy (27), is given as follows:

$$
\bar{m}_{k}=\left\{\begin{array}{cl}
\min \left\{s_{k, 1}^{2}, s_{k, 2}^{2}\right\}, & \mathcal{A}_{k} \cap \mathcal{B}_{k}=\emptyset, \mathcal{E}_{\text {max }, k}>P_{\ell, k} T_{\max } \\
\max \left\{s^{2} \mid s \in \mathcal{A}_{k} \cap \mathcal{B}_{k}\right\}, & \mathcal{A}_{k} \cap \mathcal{B}_{k} \neq \emptyset, \mathcal{E}_{\max , k}>P_{\ell, k} T_{\max } \\
\max \left\{s^{2} \mid s \in \mathcal{A}_{k}^{\prime} \cap \mathcal{B}_{k}\right\}, & \mathcal{A}_{k}^{\prime} \cap \mathcal{B}_{k} \neq \emptyset, \mathcal{E}_{\max , k} \leq P_{\ell, k} T_{\max }
\end{array}\right.
$$


provided that such $\bar{m}_{k}$ exists and is sufficiently large. Here,

$$
\begin{aligned}
& s_{k, 1}=\frac{-\zeta / v_{\max , k}+\sqrt{\zeta^{2} / v_{\max , k}^{2}+4 T_{\max }^{2} \rho / \alpha_{k}}}{2 T_{\max } \rho / \alpha_{k}}, \\
& s_{k, 2}= \frac{-\zeta w_{k}+\sqrt{\zeta^{2} w_{k}^{2}+4 t_{c} \widetilde{P}_{\mathrm{TX}, \min }(\chi)\left(\mathcal{E}_{\max , k}-P_{\ell, k} T_{\max }\right)}}{2 t_{c} \widetilde{P}_{\mathrm{TX}, \min }(\chi)}, \\
& s_{k, 3}= \min \left\{\sqrt{\left.\frac{\alpha_{k}}{\rho}, \frac{-\zeta w_{k}+\sqrt{\zeta^{2} w_{k}^{2}+4 t_{c} \widetilde{P}_{\mathrm{TX}, \min }(\chi) \mathcal{E}_{\max , k}}}{2 t_{c} \widetilde{P}_{\mathrm{TX}, \min }(\chi)}\right\}},\right. \\
& \mathcal{A}_{k}=\left\{s \mid s_{k, 2} \leq s \leq s_{k, 3}\right\}, \mathcal{A}_{k}^{\prime}=\left\{s \mid 0 \leq s \leq s_{k, 3}\right\}, \\
& \mathcal{B}_{k}=\left\{s \mid\left(\frac{\zeta P_{\ell, k}}{v_{\max , k}}+\zeta w_{k}\right) s+\left(t_{c} \widetilde{P}_{\mathrm{TX}, \min }(\chi)+\frac{\mathcal{E}_{\max , k} \rho}{\alpha_{k}}\right) s^{2}-\frac{\zeta w_{k} \rho}{\alpha_{k}} s^{3}-\frac{t_{c} \widetilde{P}_{\mathrm{TX}, \min }(\chi) \rho}{\alpha_{k}} s^{4} \leq \mathcal{E}_{\max , k}\right\} .
\end{aligned}
$$

Proof. Consider the constraints in (27) and assume that $m_{k}$ is large enough. By defining the variable $s \triangleq \sqrt{m_{k}}$ and considering two cases of $\frac{\mathcal{E}_{\max , k}-t_{c} m_{k} \widetilde{P}_{\mathrm{TX}, \min }(\chi)-w_{k} \zeta \sqrt{m_{k}}}{P_{\ell, k}} \geq T_{\max }$ and $\frac{\mathcal{E}_{\max , k}-t_{c} m_{k} \widetilde{P}_{\mathrm{TX}, \min }(\chi)-w_{k} \zeta \sqrt{m_{k}}}{P_{\ell, k}} \leq T_{\max }$ separately, we can conclude that any feasible $s \geq 0$ satisfies one of the following sets of constraints:

$$
\left\{\begin{array}{l}
\frac{T_{\max } \rho}{\alpha_{k}} s^{2}+\frac{\zeta}{v_{\max , k}} s \leq T_{\max } \\
t_{c} \widetilde{P}_{\mathrm{TX}, \min }(\chi) s^{2}+w_{k} \zeta s \leq \mathcal{E}_{\max , k}-P_{\ell, k} T_{\max }
\end{array}\right.
$$

or

$$
\left\{\begin{array}{l}
\mathcal{E}_{\max , k} \geq t_{c} \widetilde{P}_{\mathrm{TX}, \min }(\chi) s^{2}+w_{k} \zeta s \geq \mathcal{E}_{\max , k}-P_{\ell, k} T_{\max } \\
s^{2} \leq \frac{\alpha_{k}}{\rho} \\
\left(\frac{\zeta P_{\ell, k}}{v_{\max , k}}+\zeta w_{k}\right) s+\left(t_{c} \widetilde{P}_{\mathrm{TX}, \min }(\chi)+\frac{\mathcal{E}_{\max , k} \rho}{\alpha_{k}}\right) s^{2}-\frac{\zeta w_{k} \rho}{\alpha_{k}} s^{3}-\frac{t_{c} \widetilde{P}_{\mathrm{TX}, \min }(\chi) \rho}{\alpha_{k}} s^{4} \leq \mathcal{E}_{\max , k}
\end{array}\right.
$$

Note that the condition $s^{2} \leq \frac{\alpha_{k}}{\rho}$ is not necessary for the first set of constraints. First assume that $\mathcal{E}_{\max , k}>P_{\ell, k} T_{\max }$. Then, the maximum $s \geq 0$ that satisfies the first set of constraints is simply the minimum of the positive roots of $\frac{T_{\max } \rho}{\alpha_{k}} s^{2}+\frac{\zeta}{v_{\max , k}} s=T_{\max }$ and $t_{c} \widetilde{P}_{\mathrm{TX}, \min }(\chi) s^{2}+w_{k} \zeta s=\mathcal{E}_{\max , k}-P_{\ell, k} T_{\max }$, which are $s_{k, 1}$ and $s_{k, 2}$ in (29), respectively. Similarly, any $s \geq 0$ that satisfies the second set of constraints 1) must be greater than or equal to $s_{k, 2}$ and less than or equal to the minimum of $\sqrt{\alpha_{k} / \rho}$ and the positive root of $t_{c} \widetilde{P}_{\mathrm{TX}, \min }(\chi) s^{2}+w_{k} \zeta s=\mathcal{E}_{\max , k}$, which is $s_{k, 3}$ in (29), and 2) must satisfy the fourthorder polynomial inequality in the second set of constraints. Therefore, the maximum $s$ that satisfies the second set of constraints is simply the maximum $s$ in $\mathcal{A}_{k} \cap \mathcal{B}_{k}$, for $\mathcal{A}_{k}$ and $\mathcal{B}_{k}$ defined in (29). Note that all the elements of $\mathcal{A}_{k} \cap \mathcal{B}_{k}$ are necessarily greater than or equal to $\min \left\{s_{k, 1}, s_{k, 2}\right\}$. Therefore, if $\mathcal{A}_{k} \cap \mathcal{B}_{k} \neq \emptyset$, the upper bound on $s$ is the maximum element of $\mathcal{A}_{k} \cap \mathcal{B}_{k}$. However, if $\mathcal{A}_{k} \cap \mathcal{B}_{k}=\emptyset$, the upper bound on $s$ is given by $\min \left\{s_{k, 1}, s_{k, 2}\right\}$.

Now assume that $\mathcal{E}_{\max , k} \leq P_{\ell, k} T_{\max }$. In this case the first set of constraints does not hold for any $s \geq 0$ and only the second set of constraints needs to be considered. Following a similar procedure, we can conclude that the maximum $s$ possible that satisfies the second set of constraints is the maximum $s$ in $\mathcal{A}_{k}^{\prime} \cap \mathcal{B}_{k}$. This completes the proof. 
5.1.2. Average Minimum Energy Per Period Consumed to Cover a Set of POls by a Mobile Agent in the Communication-Intensive Case. Another performance metric to characterize probabilistically is the average of the minimum energy consumed in one period by the $k$ th mobile agent to feasibly cover its assigned POIs. We can directly characterize this, without having to use the chance-constrained approximation. This is given by the following theorem in case of large $m_{k}$, i.i.d. POIs and Gaussian channels (in the dB domain):

THEOREM 5.2. Assume that for any given channel and any set of POIs in the environments, the channel powers at the POIs are known by the mobile agents. Then, in the communication-intensive case, the average of the minimum energy consumed in one period by the kth mobile agent to feasibly cover its assigned $m_{k}$ POIs is given as follows:

$\mathcal{E}_{\text {min }, \text { ave }, k}=\left[\frac{P_{\ell, k}}{\left(1-m_{k} \frac{\rho}{\alpha_{k}}\right) v_{\max , k}}+w_{k}\right] \zeta \sqrt{m_{k}}+t_{c} m_{k} \operatorname{SNR}_{\mathrm{TH}} N_{0} B \int_{\mathcal{W}} \frac{\exp \left(\frac{1}{2} \bar{\sigma}^{2}(q)\right)}{10 \frac{\widetilde{G}_{\mathrm{dB}}(q)}{10}} \psi(q) d q$,

where $\bar{\sigma}(q) \triangleq \frac{\log (10)}{10} \widetilde{\sigma}(q), m_{k}<\frac{\alpha_{k}}{\rho}$, and $m_{k}$ remains large enough.

Proof. The minimum energy occurs when the stability margin is zero. Also, when the channel is assessed perfectly at the positions of the POIs, we have $P_{\mathrm{TX}, \min }\left(q_{i}, \chi\right)=$ $\frac{\mathrm{SNR}_{\mathrm{TH}} N_{0} B}{G\left(q_{i}\right)}$, for any $q_{i}$. By setting $\Delta_{k}=0$ and $P_{\mathrm{TX}, \min }\left(q_{i}, \chi\right)=\frac{\mathrm{SNR}_{\mathrm{TH}} N_{0} B}{G\left(q_{i}\right)}$ in $(14)$, we obtain

$$
\begin{aligned}
& \mathcal{E}_{\text {min }, \text { ave }, k}=\mathbb{E}\left\{\mathcal{E}_{k}^{*}\right\}= {\left[\frac{P_{\ell, k}}{\left(1-m_{k} \frac{\rho}{\alpha_{k}}\right) v_{\max , k}}+w_{k}\right] \mathbb{E}\left\{d\left(\mathcal{H}_{k}^{*}\right)\right\} } \\
&+t_{c} m_{k} \operatorname{SNR}_{\mathrm{TH}} N_{0} B \int_{\mathcal{W}} \mathbb{E}\left\{\frac{1}{G(q)} \mid q\right\} \psi(q) d q,
\end{aligned}
$$

where averaging is done over every possible distribution of the channel. The channel power $G(q)$ is log-normally distributed in the linear domain (it has a Gaussian distribution in the dB domain, with mean $\widetilde{G}_{\mathrm{dB}}(q)$ and variance $\left.\widetilde{\sigma}^{2}(q)\right)$. We then have

$$
\begin{aligned}
\mathbb{E}\left\{\frac{1}{G(q)} \mid q\right\} & =\int_{0}^{\infty} \frac{10}{\log (10) \sqrt{2 \pi} \tilde{\sigma}(q) G^{2}} \exp \left(-\frac{\left(10 \log _{10}(G)-\widetilde{G}_{\mathrm{dB}}(q)\right)^{2}}{2 \widetilde{\sigma}^{2}(q)}\right) d G \\
& =\frac{\exp \left(\frac{1}{2} \bar{\sigma}^{2}(q)\right)}{10^{\frac{\widetilde{G}_{\mathrm{dB}}(q)}{10}}} .
\end{aligned}
$$

Also, using Lemma 5.1, $\mathbb{E}\left\{d\left(\mathcal{H}_{k}^{*}\right)\right\}=\zeta \sqrt{m_{k}}$ for large $m_{k}$. By substituting $\mathbb{E}\left\{\frac{1}{G(q)} \mid q\right\}$ and $\mathbb{E}\left\{d\left(\mathcal{H}_{k}^{*}\right)\right\}$ into $(33),(32)$ is obtained.

\subsection{Probabilistic Analysis of the Dynamic Coverage Problem in the Communication-Efficient Case}

The same approach of Section 5.1 can be followed to probabilistically analyze the dynamic coverage problem in the communication-efficient case. Assume that $m_{k}$ is large. Similar to Section 5.1, to form the chance-constrained version of (17), when both the channel and the positions of the POIs are stochastic, it is sufficient to replace $\mathrm{d}\left(\mathcal{H}_{k}\right)$ with $\zeta \sqrt{m_{k}}$ and $\frac{\mathrm{SNR}_{\mathrm{TH}} N_{0} B}{G\left(\xi_{\mathrm{TX}, k}\right)}$ with $\widetilde{P}_{\mathrm{TX}, \min }(\chi)$, assuming that $m_{k}$ remains large. Similarly, it can be easily shown that there exists at least one feasible solution that satisfies the chance-constrained 
version of (17) if the following are true, for $k=1, \cdots, n$ :

1) $\widetilde{P}_{\mathrm{TX}, \min }(\chi) \leq P_{\mathrm{TX}, \max , k}$,

2) $m_{k}<\frac{\alpha_{k}}{\rho}$,

3) $\min \left\{\frac{\mathcal{E}_{\max , k}-t_{c} \widetilde{P}_{\mathrm{TX}, \min }(\chi)-w_{k} \zeta \sqrt{m_{k}}}{P_{\ell, k}}, T_{\max }\right\}\left(1-m_{k} \frac{\rho}{\alpha_{k}}\right) \geq \frac{\zeta \sqrt{m_{k}}}{v_{\max , k}}$,

where $m_{k}$ is assumed large enough. It can be seen that assuming $t_{c}$ is the same for both communication-intensive and communication-efficient cases, the conditions in (35) are less restrictive than those of $(27)$, as the mobile agents are required to communicate to the remote station once during their trajectories.

5.2.1. An Upper Bound on the Maximum Number of POls Covered by a Mobile Agent in the Communication-Efficient Case. Similar to the communication-intensive case, we use (35) to find an upper bound on the maximum number of POIs that can be assigned to each agent. Consider the following theorem:

THEOREM 5.3. Assume that for the $k$ th agent and for the given $\chi$, we have $\widetilde{P}_{\mathrm{TX}, \min }(\chi) \leq$ $P_{\mathrm{TX}, \max , k}$, where $\widetilde{P}_{\mathrm{TX}, \min }(\chi)$ is given by (25). Then, in the communication-efficient case, an upper-bound on the maximum number of POIs that can be assigned to the kth agent, to satisfy (35), is given as follows:

$$
\bar{m}_{k}=\left\{\begin{array}{cl}
\min \left\{s_{k, 1}^{2}, s_{k, 2}^{2}\right\}, & \mathcal{A}_{k} \cap \mathcal{B}_{k}=\emptyset, \mathcal{E}_{\max , k}-t_{c} \widetilde{P}_{\mathrm{TX}, \min }(\chi)>P_{\ell, k} T_{\max } \\
\max \left\{s^{2} \mid s \in \mathcal{A}_{k} \cap \mathcal{B}_{k}\right\}, & \mathcal{A}_{k} \cap \mathcal{B}_{k} \neq \emptyset, \mathcal{E}_{\max , k}-t_{c} \widetilde{P}_{\mathrm{TX}, \min }(\chi)>P_{\ell, k} T_{\max }, \\
\max \left\{s^{2} \mid s \in \mathcal{A}_{k}^{\prime} \cap \mathcal{B}_{k}\right\}, & \mathcal{A}_{k}^{\prime} \cap \mathcal{B}_{k} \neq \emptyset,
\end{array}\right.
$$

provided that such $\bar{m}_{k}$ exists and is sufficiently large. Here,

$$
\begin{aligned}
s_{k, 1} & =\frac{-\zeta / v_{\max , k}+\sqrt{\zeta^{2} / v_{\max , k}^{2}+4 T_{\max }^{2} \rho / \alpha_{k}}}{2 T_{\max } \rho / \alpha_{k}}, \\
s_{k, 2} & =\frac{\mathcal{E}_{\max , k}-t_{c} \widetilde{P}_{\mathrm{TX}, \min }(\chi)-P_{\ell, k} T_{\max }}{\zeta w_{k}}, \\
s_{k, 3} & =\min \left\{\sqrt{\left.\frac{\alpha_{k}}{\rho}, \frac{\mathcal{E}_{\max , k}-t_{c} \widetilde{P}_{\mathrm{TX}, \min }(\chi)}{\zeta w_{k}}\right\},}\right. \\
\mathcal{A}_{k} & =\left\{s \mid s_{k, 2} \leq s \leq s_{k, 3}\right\}, \mathcal{A}_{k}^{\prime}=\left\{s \mid 0 \leq s \leq s_{k, 3}\right\}, \\
\mathcal{B}_{k} & =\left\{s \mid\left(\frac{\zeta P_{\ell, k}}{v_{\max , k}}+\zeta w_{k}\right) s+\frac{\left(\mathcal{E}_{\max , k}-t_{c} \widetilde{P}_{\mathrm{TX}, \min }(\chi)\right) \rho}{\alpha_{k}} s^{2}-\frac{\zeta w_{k} \rho}{\alpha_{k}} s^{3} \leq \mathcal{E}_{\max , k}\right\} .
\end{aligned}
$$

Proof. Consider the constraints in (35) and assume that $m_{k}$ is large enough. By defining the variable $s \triangleq \sqrt{m_{k}}$ and considering two cases of $\frac{\mathcal{E}_{\max , k}-t_{c} \widetilde{P}_{\mathrm{TX}, \min }(\chi)-w_{k} \zeta \sqrt{m_{k}}}{P_{\ell, k}} \geq T_{\max }$ and $\frac{\mathcal{E}_{\max , k}-t_{c} \widetilde{P}_{\mathrm{TX}, \min }(\chi)-w_{k} \zeta \sqrt{m_{k}}}{P_{\ell, k}} \leq T_{\max }$ separately, we can conclude that any feasible $s \geq 0$ satisfies one of the following sets of constraints:

$$
\left\{\begin{array}{l}
\frac{T_{\max } \rho}{\alpha_{k}} s^{2}+\frac{\zeta}{v_{\max , k}} s \leq T_{\max } \\
s \leq \frac{\mathcal{E}_{\max , k}-t_{c} \widetilde{P}_{\mathrm{TX}, \min }(\chi)-P_{\ell, k} T_{\max }}{\zeta w_{k}}
\end{array}\right.
$$


or

$$
\left\{\begin{array}{l}
\frac{\mathcal{E}_{\mathrm{max}, k}-t_{c} \widetilde{P}_{\mathrm{TX}, \min }(\chi)}{\zeta w_{k}} \geq s \geq \frac{\mathcal{E}_{\mathrm{max}, k}-t_{c} \widetilde{P}_{\mathrm{TX}, \min }(\chi)-P_{\ell, k} T_{\max }}{\zeta w_{k}} \\
s^{2} \leq \frac{\alpha_{k}}{\rho} \\
\left(\frac{\zeta P_{\ell, k}}{v_{\max , k}}+\zeta w_{k}\right) s+\frac{\left(\mathcal{E}_{\max , k}-t_{c} \widetilde{P}_{\mathrm{TX}, \min }(\chi)\right) \rho}{\alpha_{k}} s^{2}-\frac{\zeta w_{k} \rho}{\alpha_{k}} s^{3} \leq \mathcal{E}_{\max , k}
\end{array}\right.
$$

The maximum $s$ that satisfies one of these constraints is then found using a procedure similar to that of Theorem 5.1 for two cases of $\mathcal{E}_{\max , k}-t_{c} \widetilde{P}_{\mathrm{TX}, \min }(\chi)>P_{\ell, k} T_{\max }$ and $\mathcal{E}_{\max , k}-t_{c} \widetilde{P}_{\mathrm{TX}, \min }(\chi) \leq P_{\ell, k} T_{\max }$

5.2.2. Average Minimum Energy Per Period Consumed to Cover a Set of POls by a Mobile Agent in the Communication-Efficient Case. Similar to the communication-intensive case, the average of the minimum energy consumed in one period by the $k$ th mobile agent to cover its assigned POIs in the communication-efficient case is given by the following theorem:

THEOREM 5.4. Without loss of generality, assume that $\mathcal{V}_{k}=\left\{1, \cdots, m_{k}\right\}$. Also, assume that for any given channel and any set of POIs in the environments, the channel powers at the POIs are known by the mobile agents. Then, in the communication-efficient case, the average of the minimum energy consumed in one period by the kth mobile agent to feasibly cover its assigned $m_{k}$ POIs is given as follows:

$$
\begin{aligned}
\mathcal{E}_{\text {min }, \text { ave }, k} & =\left[\frac{P_{\ell, k}}{\left(1-m_{k} \frac{\rho}{\alpha_{k}}\right) v_{\max , k}}+w_{k}\right] \zeta \sqrt{m_{k}} \\
& +t_{c} \operatorname{SNR}_{\mathrm{TH}} N_{0} B \int_{\mathcal{W}} \cdots \int_{\mathcal{W}} \int_{-\infty}^{\infty} 10^{-x / 10} \frac{\partial}{\partial x} \Upsilon\left(x, Q_{k}\right) \psi\left(q_{1}\right) \cdots \psi\left(q_{m_{k}}\right) d x d Q_{k},
\end{aligned}
$$

where $Q_{k} \triangleq\left[q_{1}^{\mathrm{T}}, \cdots, q_{m_{k}}^{\mathrm{T}}\right]^{\mathrm{T}}$ is the stacked vector of the positions of the POIs in $\mathcal{V}_{k}, m_{k}<$ $\frac{\alpha_{k}}{\rho}$,

$$
\Upsilon\left(x, Q_{k}\right) \triangleq \int_{-\infty}^{x} \cdots \int_{-\infty}^{x} \frac{\exp \left(-\frac{1}{2}\left(G_{\mathrm{dB}}-\widetilde{G}_{\mathrm{dB}}\left(Q_{k}\right)\right)^{\mathrm{T}} \widetilde{\Sigma}^{-1}\left(Q_{k}\right)\left(G_{\mathrm{dB}}-\widetilde{G}_{\mathrm{dB}}\left(Q_{k}\right)\right)\right)}{(2 \pi)^{\frac{m_{k}}{2}}\left|\widetilde{\Sigma}\left(Q_{k}\right)\right|^{\frac{1}{2}}} d G_{\mathrm{dB}}
$$

and $\widetilde{G}_{\mathrm{dB}}\left(Q_{k}\right)$ and $\widetilde{\Sigma}\left(Q_{k}\right)$ denote the mean vector and the covariance matrix of the multivariate Gaussian distribution characterizing the channel powers at the POIs in the $d B$ domain.

Proof. The proof is similar to the proof of Theorem 5.2. The minimum energy occurs when the stability margin is zero. Also, when the channel is assessed perfectly at the positions of the POIs, we have $P_{\mathrm{TX}, \min }\left(q_{i}, \chi\right)=\frac{\mathrm{SNR}_{\mathrm{TH}} N_{0} B}{G\left(q_{i}\right)}$, for any $q_{i}$. By setting $\Delta_{k}=0$ and $P_{\mathrm{TX}, \min }\left(q_{i}, \chi\right)=\frac{\mathrm{SNR}_{\mathrm{TH}} N_{0} B}{G\left(q_{i}\right)}$ in $(21)$, we obtain

$$
\begin{aligned}
\mathcal{E}_{\text {min }, \text { ave }, k} & =\mathbb{E}\left\{\mathcal{E}_{k}^{*}\right\}=\left[\frac{P_{\ell, k}}{\left(1-m_{k} \frac{\rho}{\alpha_{k}}\right) v_{\max , k}}+w_{k}\right] \mathbb{E}\left\{d\left(\mathcal{H}_{k}^{*}\right)\right\} \\
& +t_{c} \mathrm{SNR}_{\mathrm{TH}} N_{0} B \int_{\mathcal{W}} \cdots \int_{\mathcal{W}} \mathbb{E}\left\{\frac{1}{\max _{i \in \mathcal{V}_{k}} G\left(q_{i}\right)} \mid Q_{k}\right\} \psi\left(q_{1}\right) \cdots \psi\left(q_{m_{k}}\right) d Q_{k} .
\end{aligned}
$$


The joint distribution of the channel powers $G\left(q_{i}\right)$, for $i \in \mathcal{V}_{k}$, is given by a multivariate Gaussian distribution with mean vector $\widetilde{G}_{\mathrm{dB}}\left(Q_{k}\right)$ and covariance matrix $\widetilde{\Sigma}\left(Q_{k}\right)$, in the $\mathrm{dB}$ domain. Let us define $G_{\max } \triangleq \max _{i \in \mathcal{V}_{k}} G_{\mathrm{dB}}\left(q_{i}\right)$. Then, the cumulative density function (cdf) of $G_{\max }$ is given as follows:

$$
\begin{aligned}
\mathbb{P}\left\{G_{\max } \leq x\right\} & =\int_{-\infty}^{x} \cdots \int_{-\infty}^{x} \frac{\exp \left(-\frac{1}{2}\left(G_{\mathrm{dB}}-\widetilde{G}_{\mathrm{dB}}\left(Q_{k}\right)\right)^{\mathrm{T}} \widetilde{\Sigma}^{-1}\left(Q_{k}\right)\left(G_{\mathrm{dB}}-\widetilde{G}_{\mathrm{dB}}\left(Q_{k}\right)\right)\right)}{(2 \pi)^{\frac{m_{k}}{2}}\left|\widetilde{\Sigma}\left(Q_{k}\right)\right|^{\frac{1}{2}}} d G_{\mathrm{dB}} \\
& =\Upsilon\left(x, Q_{k}\right) .
\end{aligned}
$$

We therefore have

$$
\mathbb{E}\left\{\frac{1}{\max _{i \in \mathcal{V}_{k}} G\left(q_{i}\right)} \mid Q_{k}\right\}=\int_{-\infty}^{\infty} 10^{-x / 10} \frac{\partial}{\partial x} \Upsilon\left(x, Q_{k}\right) d x .
$$

Also, using Lemma 5.1, $\mathbb{E}\left\{d\left(\mathcal{H}_{k}^{*}\right)\right\}=\zeta \sqrt{m_{k}}$ for large $m_{k}$. By substituting $\mathbb{E}\left\{\frac{1}{G(q)} \mid q\right\}$ and $\mathbb{E}\left\{d\left(\mathcal{H}_{k}^{*}\right)\right\}$ into (42), (40) is obtained.

Note that the average of the minimum energy per period in the communication-efficient case is more complicated than the one derived in the communication-intensive case, due to the dependency of the joint pdf of the channel powers at the POIs. Another important difference is that the communication part in $\mathcal{E}_{\text {min,ave, } k}$ is a decreasing function of $m_{k}$ in the communication-efficient case, while it is an increasing function of $m_{k}$ in the communicationintensive case. This can be explained using the fact that in a given environment by increasing the number of POIs, the chance of finding a larger channel power will also increase. This decreases the transmission power required for connectivity in the communication-efficient case. This is explained in more details in the next section.

\section{SIMULATION RESULTS}

In this section, we present our simulation results for the dynamic coverage of a time-varying environment using the proposed framework. The simulation environment was implemented in $\mathrm{C}++$ and MATLAB. To solve the MILPs, we used IBM ILOG CPLEX Optimization Studio v12.2.

Fig. 3 shows the result of applying the proposed dynamic coverage framework to cover 24 POIs using 3 mobile agents, in a $200 \mathrm{~m}$ by $200 \mathrm{~m}$ workspace. The wireless channel between the mobile agents and the remote station is generated using our probabilistic channel simulator, which can generate path loss, shadowing and multipath fading with realistic spatial correlations. A detailed description of this channel simulator can be found in [Mostofi et al. 2009; Gonzalez-Ruiz et al. 2011]. The 3D plot of the channel power over the workspace is shown in Fig. 2. In this example, the shadowing component of the channel is log-normally distributed in the linear domain (has a zero-mean Gaussian distribution in the dB domain). The multipath fading component is also Rician-distributed in the linear domain. The remote station is located at position $q_{b}=(-80,80,0.5) \mathrm{m}$. The following channel parameters are also used: $K_{\mathrm{dB}}=-5 \mathrm{~dB}, n_{\mathrm{PL}}=2, \vartheta=5 \mathrm{~dB}, \beta=30 \mathrm{~m}$ and $\omega=2 \mathrm{~dB}$. See Appendix A for the descriptions of the channel parameters and the distributions of the shadowing and multipath components. The rest of the parameters are as follows: $\mathrm{SNR}_{\mathrm{TH}}=25 \mathrm{~dB}, B N_{0}=-90$ $\mathrm{dB}, \alpha_{i, k}=100, T_{\max }=6000 \mathrm{~s}, \mathcal{E}_{\max , k}=40 \mathrm{~J}, v_{\max , k}=0.1 \mathrm{~m} / \mathrm{s}, P_{\mathrm{TX}, \max , k}=800 \mathrm{~mW}$, $P_{\ell, m, k}=0.1 \mathrm{~mW}, P_{\ell, s, k}=0.2 \mathrm{~mW}, w_{k}=0.1 \mathrm{~J} / \mathrm{m}$ (corresponding to a small-size light-load robot) and $\Delta_{k}=0$, for $i=1, \cdots, 24$ and $k=1,2,3$. Furthermore, we set $\varrho_{k}=\frac{1}{3}$, for $k=1,2,3$, and $t_{c}=20 \mathrm{~s}$ in both communication-intensive and communication-efficient cases. Note that the value of $t_{c}$ is small enough such that communication can be done while stopping at the positions of the POIs (or while being very close to them). 


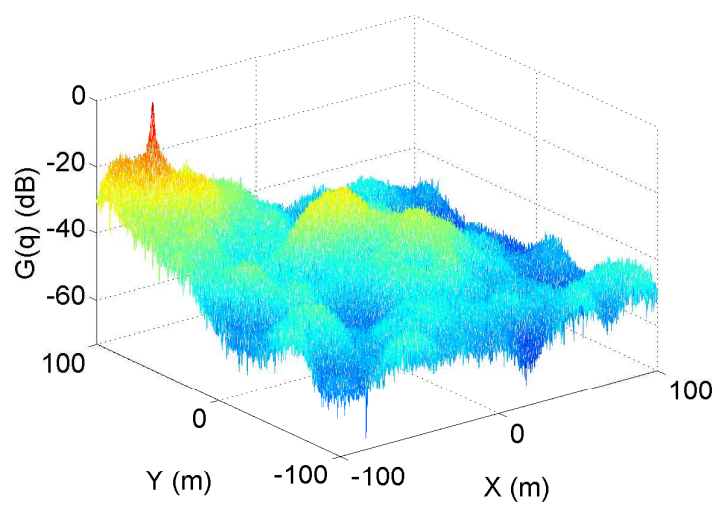

Fig. 2: The 3D plot of the channel power $G(q)$ over the workspace of Fig. 3.

Fig. 3 (left) and Fig. 3 (right) show the optimal trajectories of the mobile agents in the communication-intensive and communication-efficient cases respectively and for the case of known channel. The optimal communication points for each mobile agent in the communication-efficient case is also specified by a circle in Fig. 3 (right). The values of $\rho_{i}$ and $G\left(q_{i}\right)$ for all the POIs are listed in Table II. The optimal stop times at all the POIs, in both communication-intensive and communication-efficient cases, are listed in Table III. The optimal period, optimal total energy per period, optimal motion energy per period and optimal communication energy per period, in both communication-intensive and communication-efficient cases and for all the mobile agents, are also listed in Table IV.

In can be seen that the optimal period and the optimal motion energy per period are larger for longer routes, as expected. The optimal communication energy per period, on the other hand, is a function of channel qualities at the POIs. For instance, in the communicationintensive case, Agent 2 (dashed-blue trajectory) is assigned to 5 POIs only. However, the optimal communication energy per period is the largest for this agent. This is due to the fact that the POIs assigned to this agent experience the lowest channel qualities among the POIs, as can be seen from Table II. On the other hand, Agent 1 (solid-red trajectory) consumes the minimum communication energy in the communication-intensive case as its assigned POIs experience highest channel qualities. The same discussion applies to the optimal motion and communication energies in the communication-efficient case.

From Table IV, one can also see that the communication energy per period in the communication-efficient case is much less than the one in the communication-intensive case, as expected. ${ }^{11}$ Furthermore, it can be confirmed that the optimal communication point for each mobile agent in the communication-efficient case is the POI that experiences the maximum channel power among all the POIs assigned to that agent. It is worth mentioning that in this example the mobile agents are identical. Therefore, we can alternatively assign any mobile agent to any partition, in both communication-intensive and communication-efficient cases, without changing the optimal solution. Another important note is that for a given $\mathcal{E}_{\max , k}$, the communication-efficient case imposes less constraint on the motion, since less communication energy is consumed as compared to the communication-intensive case (same $t_{c}$ is used for both cases). Note that the optimal partition found for the communicationefficient case cannot be used for the communication-intensive case as it violates the constraint on the total energy per period.

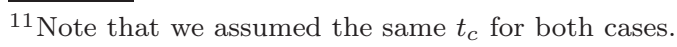




\begin{tabular}{|c|c|c||c|c|c||c|c|c|}
\hline POI & $\rho_{i}$ & $G\left(q_{i}\right)(\mathrm{dB})$ & $\mathrm{POI}$ & $\rho_{i}$ & $G\left(q_{i}\right)(\mathrm{dB})$ & $\mathrm{POI}$ & $\rho_{i}$ & $G\left(q_{i}\right)(\mathrm{dB})$ \\
\hline 1 & 2.01 & -48.3 & 9 & 2.89 & -42.8 & 17 & 2.38 & -43.2 \\
\hline 2 & 1.47 & -49.3 & 10 & 1.92 & -62.7 & 18 & 1.26 & -30.3 \\
\hline 3 & 1.23 & -46.3 & 11 & 1.78 & -51 & 19 & 1.74 & -40.1 \\
\hline 4 & 2.84 & -45.9 & 12 & 1.54 & -56.4 & 20 & 2.78 & -43.7 \\
\hline 5 & 2.91 & -37.1 & 13 & 2.97 & -51.4 & 21 & 1.34 & -35.5 \\
\hline 6 & 2.71 & -46.5 & 14 & 1.59 & -53.8 & 22 & 2.85 & -54.1 \\
\hline 7 & 1.19 & -45.2 & 15 & 1.78 & -49.4 & 23 & 2.42 & -42.9 \\
\hline 8 & 1.71 & -39.8 & 16 & 1.39 & -41.7 & 24 & 2.07 & -34.9 \\
\hline
\end{tabular}

Table II: The value of $\rho_{i}$ and $G\left(q_{i}\right)$ at the POIs in Fig. 3.

\begin{tabular}{|c|c||c|c|c|c||c|c|}
\hline \multicolumn{9}{|c|}{ Communication-Intensive Case } & \multicolumn{3}{|c|}{ Communication-Efficient Case } \\
\hline POI & $t_{i, k}^{*}$ & POI & $t_{i, k}^{*}$ & POI & $t_{i, k}^{*}$ & POI & $t_{i, k}^{*}$ \\
\hline 1 & $63.3 \mathrm{~s}$ & 13 & $93.5 \mathrm{~s}$ & 1 & $107 \mathrm{~s}$ & 13 & $158 \mathrm{~s}$ \\
\hline 2 & $46.1 \mathrm{~s}$ & 14 & $50 \mathrm{~s}$ & 2 & $77.6 \mathrm{~s}$ & 14 & $84.2 \mathrm{~s}$ \\
\hline 3 & $54.4 \mathrm{~s}$ & 15 & $78.5 \mathrm{~s}$ & 3 & $49.9 \mathrm{~s}$ & 15 & $72 \mathrm{~s}$ \\
\hline 4 & $50.2 \mathrm{~s}$ & 16 & $24.4 \mathrm{~s}$ & 4 & $151 \mathrm{~s}$ & 16 & $56.1 \mathrm{~s}$ \\
\hline 5 & $91.6 \mathrm{~s}$ & 17 & $105 \mathrm{~s}$ & 5 & $154 \mathrm{~s}$ & 17 & $96.2 \mathrm{~s}$ \\
\hline 6 & $47.8 \mathrm{~s}$ & 18 & $55.6 \mathrm{~s}$ & 6 & $144 \mathrm{~s}$ & 18 & $6.2 \mathrm{~s}$ \\
\hline 7 & $52.6 \mathrm{~s}$ & 19 & $76.8 \mathrm{~s}$ & 7 & $48.2 \mathrm{~s}$ & 19 & $8.56 \mathrm{~s}$ \\
\hline 8 & $75.6 \mathrm{~s}$ & 20 & $123 \mathrm{~s}$ & 8 & $69.4 \mathrm{~s}$ & 20 & $113 \mathrm{~s}$ \\
\hline 9 & $128 \mathrm{~s}$ & 21 & $42.1 \mathrm{~s}$ & 9 & $117 \mathrm{~s}$ & 21 & $70.9 \mathrm{~s}$ \\
\hline 10 & $33.8 \mathrm{~s}$ & 22 & $89.7 \mathrm{~s}$ & 10 & $101 \mathrm{~s}$ & 22 & $151 \mathrm{~s}$ \\
\hline 11 & $56 \mathrm{~s}$ & 23 & $76 \mathrm{~s}$ & 11 & $94.4 \mathrm{~s}$ & 23 & $128 \mathrm{~s}$ \\
\hline 12 & $27.1 \mathrm{~s}$ & 24 & $91.6 \mathrm{~s}$ & 12 & $81.4 \mathrm{~s}$ & 24 & $84 \mathrm{~s}$ \\
\hline
\end{tabular}

Table III: The optimal stop times at all the POIs in Fig. 3 in both communication-intensive and communication-efficient cases.

\begin{tabular}{|c|c|c|c|c||c|c|c|c|c|}
\hline \multicolumn{4}{|c||}{ Communication-Intensive Case } & \multicolumn{4}{c|}{ Communication-Efficient Case } \\
\hline$k$ & $T_{k}^{*}$ & $\mathcal{E}_{k}^{*}$ & $\mathcal{E}_{m, k}^{*}$ & $\mathcal{E}_{\mathrm{TX}, k}^{*}$ & $k$ & $T_{k}^{*}$ & $\mathcal{E}_{k}^{*}$ & $\mathcal{E}_{m, k}^{*}$ & $\mathcal{E}_{\mathrm{TX}, k}^{*}$ \\
\hline 1 & $4414 \mathrm{~s}$ & $37.85 \mathrm{~J}$ & $36.26 \mathrm{~J}$ & $1.587 \mathrm{~J}$ & 1 & $4050 \mathrm{~s}$ & $33.94 \mathrm{~J}$ & $33.92 \mathrm{~J}$ & $0.01939 \mathrm{~J}$ \\
\hline 2 & $1764 \mathrm{~s}$ & $31.14 \mathrm{~J}$ & $16 \mathrm{~J}$ & $15.14 \mathrm{~J}$ & 2 & $5298 \mathrm{~s}$ & $38.67 \mathrm{~J}$ & $38.64 \mathrm{~J}$ & $0.02229 \mathrm{~J}$ \\
\hline 3 & $3144 \mathrm{~s}$ & $31.66 \mathrm{~J}$ & $25.74 \mathrm{~J}$ & $5.924 \mathrm{~J}$ & 3 & $492.1 \mathrm{~s}$ & $4.831 \mathrm{~J}$ & $4.824 \mathrm{~J}$ & $0.006781 \mathrm{~J}$ \\
\hline
\end{tabular}

Table IV: The optimal period, optimal total energy per period, optimal motion energy per period and optimal communication energy per period in both communication-intensive and communication-efficient cases and for all the mobile agents in Fig. 3.

Fig. 4 (left) and Fig. 4 (right) show the plots of $\Psi_{i}(t)$ at the remote station for one sample POI in Fig. 3 (POI \#4), in communication-intensive and communication-efficient cases respectively. In the communication-efficient case, the plot of $\Phi_{i}(t)$ is also shown (note that in the communication-intensive case $\Psi_{i}(t)$ is identical to $\left.\Phi_{i}(t)\right)$. Without loss of generality, in both figures we assume that at $t=0$ the agent starts at POI 4 . In the communicationefficient case, we also assume that communication happens at the end of visiting the POI that is selected as the optimal communication point (POI 21 in this case). In can be seen that $\Psi_{i}(t)$ remains bounded at the remote station in both cases. Similar plots can also be obtained for other POIs in Fig. 3.

Note that for a fixed $\mathcal{V}_{k}$, the maximum value of $\Psi_{i}(t)$ for any $i \in \mathcal{V}_{k}$ is larger in the communication-efficient case, as compared to the communication-intensive case. This is due to the fact that in the communication-efficient case, there is generally a delay in reporting 

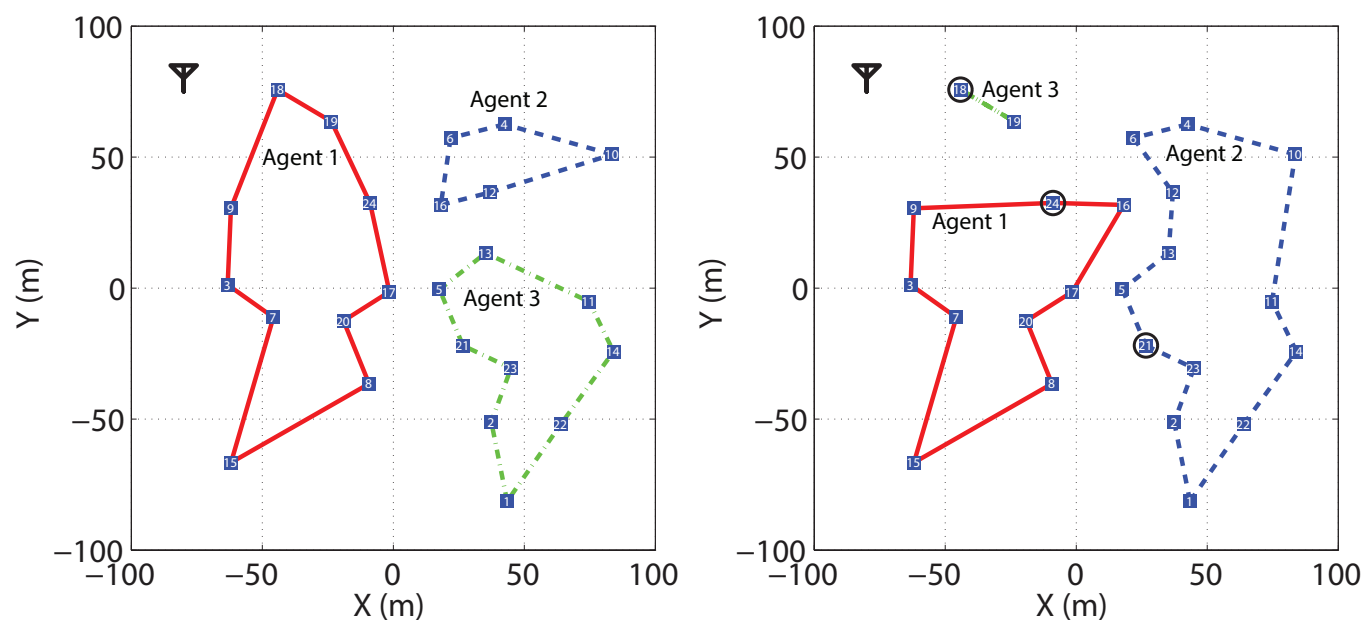

Fig. 3: The optimal trajectories of the mobile agents in the communication-intensive (left) and communication-efficient (right) cases for the case of known channel. The solid red, dashed blue and dotdashed green lines correspond to the trajectories of Agent 1,2 and 3 respectively. The location of the remote station is denoted at the top left corner of the figures. The optimal communication points for each mobile agent in the communication-efficient case is also specified by a circle in the right figure.

the observation of each POI, which results in a non-zero minimum for $\Psi_{i}(t)$. However, the communication-efficient case can stabilize the dynamic coverage task with less constraints on the connectivity.
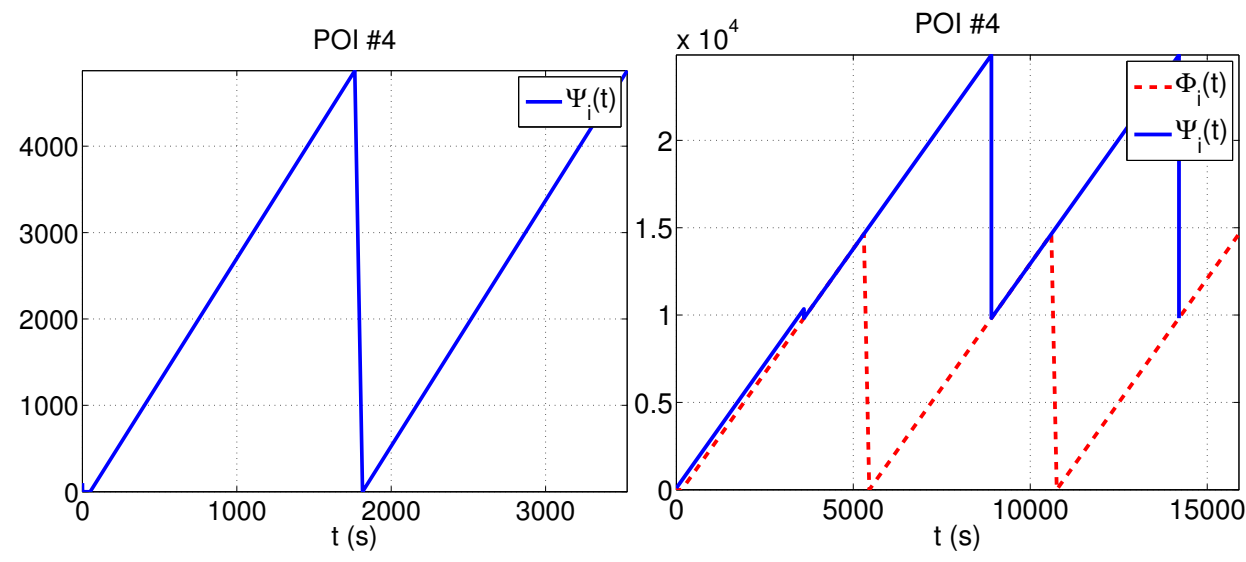

Fig. 4: The plot of $\Psi_{i}(t)$ at the remote station for POI \#4 in Fig. 3 in communication-intensive (left) and communication-efficient (right) cases. In the communication-efficient case, the plot of $\Phi_{i}(t)$ at the mobile agent is also provided.

Next, consider the case where the channel powers at the POIs are not known and are assessed probabilistically. Assume the same workspace and channel of Fig. 3. In order to show our results more clearly, assume that only one mobile agent is used to cover the POIs. The system parameters are taken to be the same as the previous case, except we have $\alpha_{i}=200, T_{\max }=12000 \mathrm{~s}, \mathcal{E}_{\max }=200 \mathrm{~J}$, and $\Delta=2000$ in this case. Note that we dropped the dependency of the system parameters on $k$, as we have only one mobile agent in this case. In this example, we assume that the channel is assessed using $0.5 \%$ of 


\begin{tabular}{|c|c|c|c||c|c|c|c|}
\hline POI & $t_{i}^{*}$ & $P_{\mathrm{TX}, \min }\left(q_{i}, \chi\right)$ & $\frac{\mathrm{SNR}_{\mathrm{TH}} N_{0} B}{G\left(q_{i}\right)}$ & $\mathrm{POI}$ & $t_{i}^{*}$ & $P_{\mathrm{TX}, \min }\left(q_{i}, \chi\right)$ & $\frac{\mathrm{SNR}_{\mathrm{TH}} N_{0} B}{G\left(q_{i}\right.}$ \\
\hline 1 & $113.13 \mathrm{~s}$ & $67.259 \mathrm{~mW}$ & $21.316 \mathrm{~mW}$ & 13 & $162.38 \mathrm{~s}$ & $127.25 \mathrm{~mW}$ & $43.702 \mathrm{~mW}$ \\
\hline 2 & $85.084 \mathrm{~s}$ & $122.72 \mathrm{~mW}$ & $26.615 \mathrm{~mW}$ & 14 & $91.422 \mathrm{~s}$ & $200.61 \mathrm{~mW}$ & $74.99 \mathrm{~mW}$ \\
\hline 3 & $73.151 \mathrm{~s}$ & $44.735 \mathrm{~mW}$ & $13.532 \mathrm{~mW}$ & 15 & $101.09 \mathrm{~s}$ & $125.84 \mathrm{~mW}$ & $27.739 \mathrm{~mW}$ \\
\hline 4 & $155.76 \mathrm{~s}$ & $45.637 \mathrm{~mW}$ & $12.171 \mathrm{~mW}$ & 16 & $81.005 \mathrm{~s}$ & $36.209 \mathrm{~mW}$ & $4.7053 \mathrm{~mW}$ \\
\hline 5 & $159.26 \mathrm{~s}$ & $4.1431 \mathrm{~mW}$ & $1.6186 \mathrm{~mW}$ & 17 & $131.75 \mathrm{~s}$ & $16.905 \mathrm{~mW}$ & $6.5547 \mathrm{~mW}$ \\
\hline 6 & $148.9 \mathrm{~s}$ & $71.488 \mathrm{~mW}$ & $14.09 \mathrm{~mW}$ & 18 & $74.563 \mathrm{~s}$ & $1.338 \mathrm{~mW}$ & $0.33904 \mathrm{~mW}$ \\
\hline 7 & $71.034 \mathrm{~s}$ & $49.583 \mathrm{~mW}$ & $10.496 \mathrm{~mW}$ & 19 & $99.129 \mathrm{~s}$ & $12.229 \mathrm{~mW}$ & $3.206 \mathrm{~mW}$ \\
\hline 8 & $97.799 \mathrm{~s}$ & $18.894 \mathrm{~mW}$ & $2.9919 \mathrm{~mW}$ & 20 & $152.51 \mathrm{~s}$ & $22.329 \mathrm{~mW}$ & $7.4526 \mathrm{~mW}$ \\
\hline 9 & $158.17 \mathrm{~s}$ & $28.176 \mathrm{~mW}$ & $6.0823 \mathrm{~mW}$ & 21 & $78.6 \mathrm{~s}$ & $7.8333 \mathrm{~mW}$ & $1.1143 \mathrm{~mW}$ \\
\hline 10 & $108.17 \mathrm{~s}$ & $774.54 \mathrm{~mW}$ & $589.35 \mathrm{~mW}$ & 22 & $156.13 \mathrm{~s}$ & $224.98 \mathrm{~mW}$ & $80.994 \mathrm{~mW}$ \\
\hline 11 & $101.33 \mathrm{~s}$ & $165.64 \mathrm{~mW}$ & $39.749 \mathrm{~mW}$ & 23 & $133.92 \mathrm{~s}$ & $41.428 \mathrm{~mW}$ & $6.118 \mathrm{~mW}$ \\
\hline 12 & $88.73 \mathrm{~s}$ & $185.93 \mathrm{~mW}$ & $136.71 \mathrm{~mW}$ & 24 & $116.3 \mathrm{~s}$ & $2.1681 \mathrm{~mW}$ & $0.96975 \mathrm{~mW}$ \\
\hline
\end{tabular}

Table V: The optimal stop times (for both communication-intensive and communication-efficient cases), and the values of $\frac{\operatorname{SNR}_{\mathrm{TH}} N_{0} B}{G\left(q_{i}\right)}$ and $P_{\mathrm{TX}, \min }\left(q_{i}, \chi\right)$ for all the POIs in Fig. 5.

the total channel power samples (804 samples in a $401 \times 401$ grid), which are assumed to be randomly collected during an offline survey of the channel (see Appendix A for a discussion on our probabilistic channel assessment framework). Fig. 5 (left) compares the estimated and actual channel powers at the positions of the POIs. Fig. 5 (right) then shows the optimal trajectory of the mobile agent in both communication-intensive and communication-efficient cases respectively. Note that, as proved by Theorems 3.1 and 4.1, the optimal trajectory in case of a single mobile agent is the minimum-length Hamiltonian cycle, in both communication-intensive and communication efficient cases. The optimal communication point in the communication efficient case, i.e., the POI with the smallest $P_{\mathrm{TX}, \min }\left(q_{i}, \chi\right)$ (see Section 4.1), is also specified by a circle in Fig. 5 (right). Table $\mathrm{V}$ lists the optimal stop times and the value of $P_{\mathrm{TX}, \min }\left(q_{i}, \chi\right)$ at all the POIs. For the sake of comparison, this table also shows the minimum required transmit power for the case of known channel powers, i.e., $\frac{\mathrm{SNR}_{\mathrm{TH}} N_{0} B}{G\left(q_{i}\right)}$, for all the POIs. The optimal period, optimal total energy per period, optimal motion energy per period and optimal communication energy per period, estimated based on the assessed channel in both communication-intensive and communication-efficient cases, are listed in Table VI. ${ }^{12}$ In this example, we set $\chi=0.95$. From Table $\mathrm{V}$, one can see that $P_{\mathrm{TX}, \min }\left(q_{i}, \chi\right)$ is larger than the minimum required transmit power in case of known channel power, i.e., $\frac{\mathrm{SNR}_{\mathrm{TH}} N_{0} B}{G\left(q_{i}\right)}$, at each POI. This is to guarantee that, based on the variance of channel estimation, the probability of connectivity is larger than $\chi$. Note that in Table V, the optimal periods are the same for both communicationintensive and communication-efficient cases, since we only have one mobile agent. Also, as can be seen from Table $\mathrm{V}$, the estimated optimal communication energy per period and, as a direct result, the estimated optimal total energy per period is larger in the communicationintensive case, as expected ( $t_{c}$ is taken to be the same for both cases).

Fig. 6 (left) and Fig. 6 (right) show the plots of $\Psi_{i}(t)$ at the remote station for two sample POIs in Fig. 5 (POI \#4 and POI \#10). For the sake of comparison, each figure shows the plots of $\Psi_{i}(t)$ at the remote station for three cases: communication-intensive, communication-efficient and communication-unaware. By communication-unaware we mean the case where the same transmission power is used at all the POIs, without adapt-

\footnotetext{
${ }^{12}$ The calculated energy values are based on assuming that the mobile agent will use the a priori found optimal channel powers, based on the probabilistic channel assessment at the POIs. Alternatively, the agent can measure the channel at the POIs after deployment and better adapt its transmission powers at the POIs.
} 

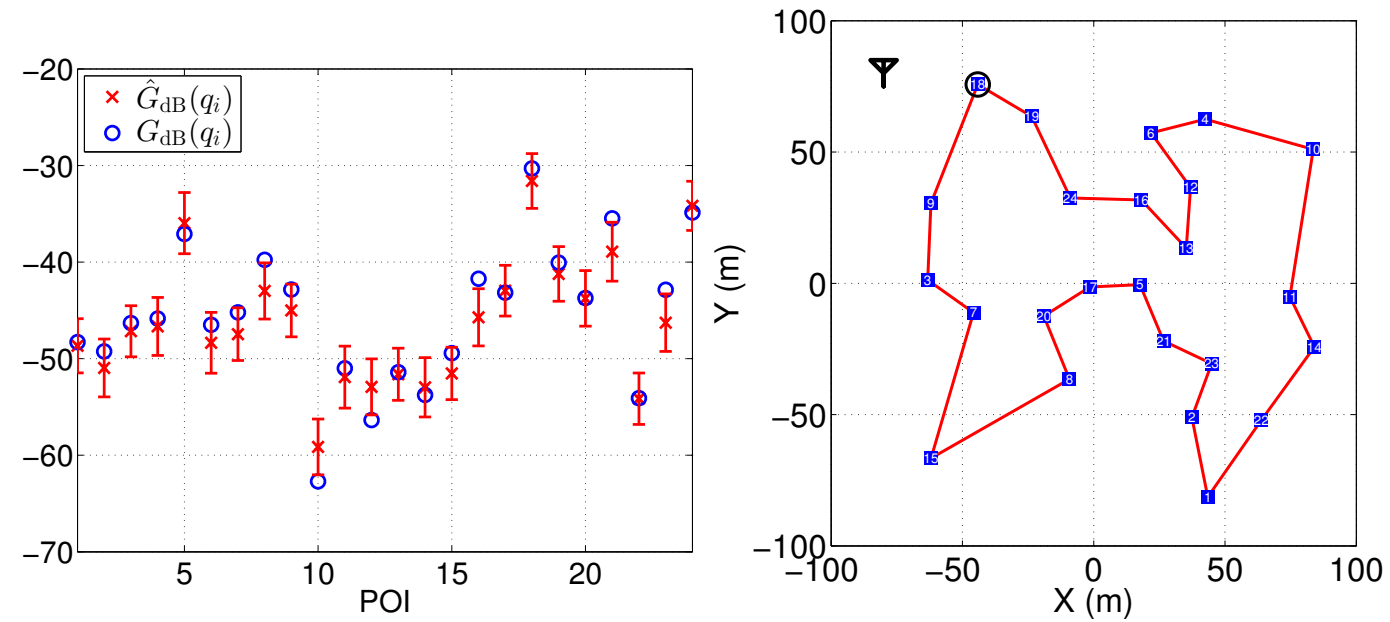

Fig. 5: The comparison of the estimated and real channel powers at the POIs (left) and the optimal trajectory of one mobile agent in both communication-intensive and communication-efficient cases and for the case of unknown channel powers (right). The location of the remote station is denoted at the top left corner of the right figure. The optimal communication point for the mobile agent in the communicationefficient case is also specified by a circle in the right figure. It can be seen that the optimal trajectory is the Hamiltonian cycle over the set of POIs.

\begin{tabular}{|c|c|c|c||c|c|c|c|}
\hline \multicolumn{4}{|c||}{ Communication-Intensive Case } & \multicolumn{4}{c|}{ Communication-Efficient Case } \\
\hline$T^{*}$ & $\mathcal{E}^{*}$ & $\mathcal{E}_{m}^{*}$ & $\mathcal{E}_{\mathrm{TX}}^{*}$ & $T^{*}$ & $\mathcal{E}^{*}$ & $\mathcal{E}_{m}^{*}$ & $\mathcal{E}_{\mathrm{TX}}^{*}$ \\
\hline $10250 \mathrm{~s}$ & $124.36 \mathrm{~J}$ & $76.403 \mathrm{~J}$ & $47.957 \mathrm{~J}$ & $10250 \mathrm{~s}$ & $76.43 \mathrm{~J}$ & $76.403 \mathrm{~J}$ & $0.02676 \mathrm{~J}$ \\
\hline
\end{tabular}

Table VI: The optimal period, optimal total energy per period, optimal motion energy per period and optimal communication energy per period, estimated based on the assessed channel in both communicationintensive and communication-efficient cases and for the mobile agent of Fig. 5. Note that the dependency on $k$ has been dropped as there is one mobile agent in this case.

ing to channel powers. To have a fair comparison, we set this fixed communication energy budget to be the same as the estimated optimal communication energy found in the communication-intensive case $(47.9573 \mathrm{~J})$. The transmission power at every POI is then fixed to $\frac{1}{m} \sum_{i \in \mathcal{V}} P_{\mathrm{TX}, \min }\left(q_{i}, \chi\right)=99.91 \mathrm{~mW}$ in this case. It can be seen that in communicationintensive and communication-efficient cases, both of the POIs could be stably covered. The communication-unaware case, however, could stabilize the coverage of only one POI (POI \#4). This is due to the fact that the mobile agent is not connected to the remote station at the position of POI \#10 in the communication-unaware case. This has been explained visually in Fig. 7. This figure shows the positions of the POIs superimposed on the connectivity map to the remote station for the communication-unaware case, assuming that the fixed transmission power of $99.91 \mathrm{~mW}$ is used. It can be seen that POI \#10 and POI \#12 are disconnected, resulting in an unstable $\Psi_{i}(t)$ at these points.

Note that after solving the proposed MILP to find the feasible stabilizing policy in this specific example, all the POIs in the communication-intensive case or the optimal communication point in the communication-efficient case are connected, although the channel is assessed probabilistically. This may or may not be the case in general, depending on the quality of the channel assessment, especially the power of the multipath fading component of the channel. Since multipath fading is not predictable using sparse sampling of the channel, one expects that by increasing the power of multipath fading the number of POIs that can be covered decreases. This is shown in Fig. 8. Fig. 8 (left) shows the percentage of the 

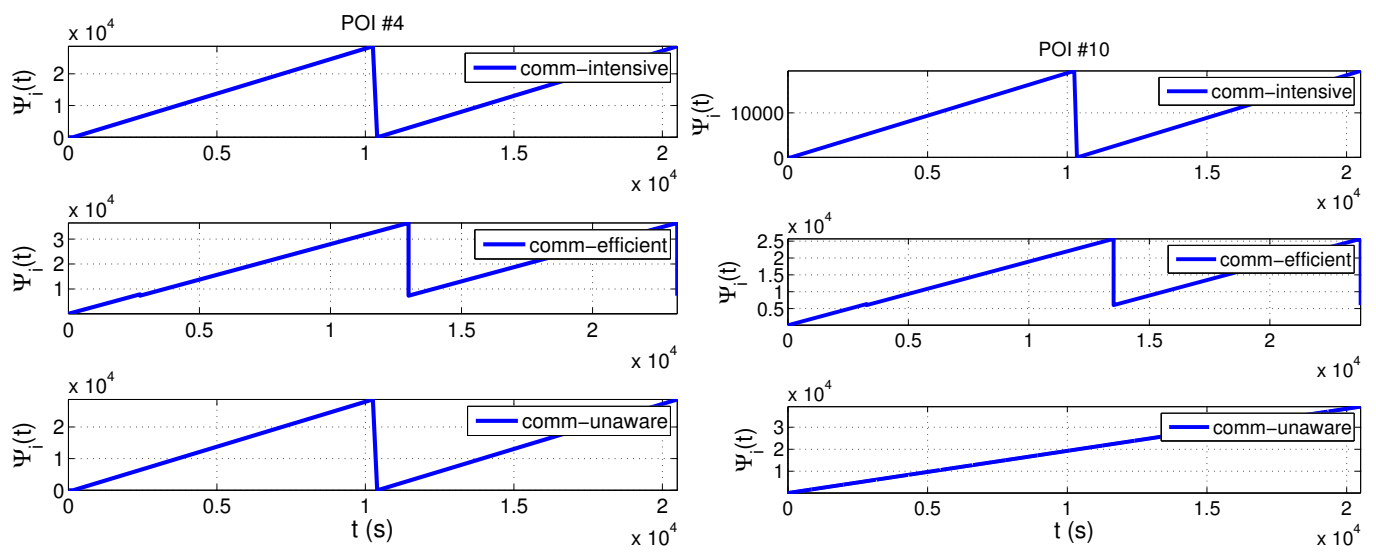

Fig. 6: The plots of $\Psi_{i}(t)$ at the remote station for POI \#4 (left) and POI \#10 (right) in Fig. 5. These figures compare the time evolution of $\Psi_{i}(t)$ in communication-intensive, communication-efficient and communication-unaware cases.

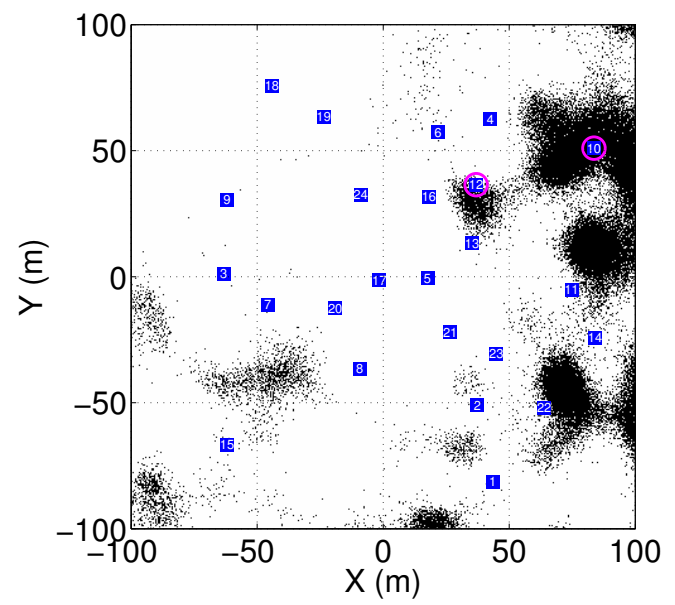

Fig. 7: Positions of the POIs superimposed on the connectivity map to the remote station, assuming that the fixed transmission power $\frac{1}{m} \sum_{i \in \mathcal{V}} P_{\mathrm{TX}, \min }\left(q_{i}, \chi\right)$ is used in the communication-unaware case. The disconnected POIs are circled on the figure.

connected POIs in Fig. 5 that can be covered by the mobile agent in the communicationintensive case, as a function of square root of multipath fading power, $\omega$. The figure also shows the results for the non-adaptive communication-unaware case. The communication energy per period in the communication-intensive case is calculated for $\chi=0.95$. The same energy budget is then used in the communication-unaware case, where the power is uniformly distributed among all the POIs. The results are averaged for 500 different realizations of the channel. In this example, the path loss and shadowing components are kept fixed and only the multipath fading component is regenerated in each realization. Similar to Fig. 5, the channel is assessed using $0.5 \%$ of the total channel power samples. Interesting results can be observed. First, it can be seen that the percentage of the POIs that can be covered in the communication-intensive case decreases as the power of multipath fading increases. Second, for a fixed multipath power, the percentage of POIs, covered in the communicationunaware case, is lower than the communication-intensive case, unless multipath power is very large. Third, for a very large multipath power, the percentage of the POIs covered 
by the communication-intensive case converges to that of the communication-unaware case. These results can be justified as follows:

As the multipath fading power increases, the quality of channel assessment degrades considerably. Although the optimal energy allocated for communication, i.e., $\sum_{i \in \mathcal{V}} P_{\mathrm{TX}, \min }\left(q_{i}, \chi\right)$, also increases as the power of multipath fading increases, the overall number of connected POIs decreases in the communication-intensive case. The increase in the optimal energy allocated for communication, however, results in an opposite effect in the communication-unaware case. Since the power is distributed uniformly among all the POIs in this case, the increase in the allocated transmission power, along with more randomness in channel variations, increases the chance of connectivity at the POIs. Finally, for very large multipath fading power, adaptation of the transmission power in the communication-intensive case is not effective anymore as channel becomes unpredictable and channel assessment can be prone to errors. This can result in almost the same percentage (or possibly worse) of connected POIs as compared to the communication-unaware case. Note that we used Rician multipath fading in this example. Therefore, the maximum possible multipath fading power in the $\mathrm{dB}$ domain is 26.1 (for $\omega=5.1195 \mathrm{~dB}$ ), which corresponds to the case when Rician distribution becomes a Raleigh distribution [Goldsmith 2005; Gonzalez-Ruiz et al. 2011]. ${ }^{13}$ To get a better idea about how the channel looks like for very small and very large multipath fading powers, Fig. 9 shows two sample channels with $\omega=0.8730 \mathrm{~dB}$ (left) and $\omega=5.0941 \mathrm{~dB}$ (right). The Rician $K$-parameter (the ratio of the power of the non-multipath component to that of the multipath component [Goldsmith 2005]) is equal to 50 for the left figure and 0.2 for the right one.

The probability of connectivity of the optimal transmission point found in the communication-efficient case also presents a similar behavior. Fig. 8 (middle) shows the plot of the probability of connectivity of the optimal transmission point in the communicationefficient case, as a function of $\omega$. The result is averaged over 500 realizations of the channel. Similar to the communication-intensive case, it can be seen that the probability of connectivity decreases as multipath power increases. Finally, Fig. 8 (right) shows the total optimal communication energy as a function of $\omega$ for both communication-intensive and communication-efficient cases. As expected, the energy increases as the multipath power increases.
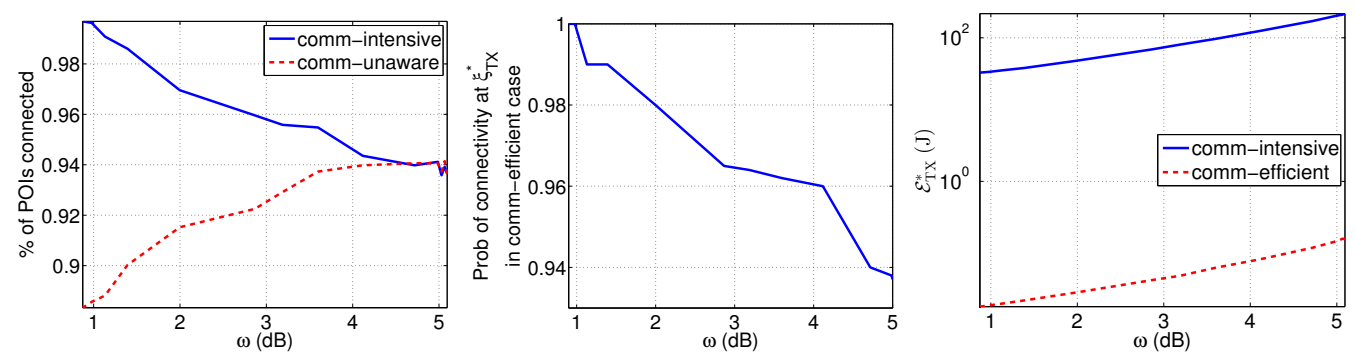

Fig. 8: The percentage of the POIs that can be covered by the mobile agent of Fig. 5 in the communicationintensive case (left), the probability of connectivity of the optimal transmission point in the communicationefficient case (middle), and the total optimal communication energy (right) as a function of $\omega$.

Finally, in order to confirm the probabilistic analysis of Section 5 for a large number of POIs, Fig. 10 compares the actual and theoretical average minimum total energy per period, consumed to cover a set of POIs by one mobile agent, as a function of the number of POIs. Fig. 11 (left) and Fig. 11 (right) also show the average minimum communication energy

\footnotetext{
${ }^{13}$ Note that the average of multipath fading term introduced in Appendix A is one in the linear domain.
} Thus, there is only one variable to work with for the Rician distribution. 

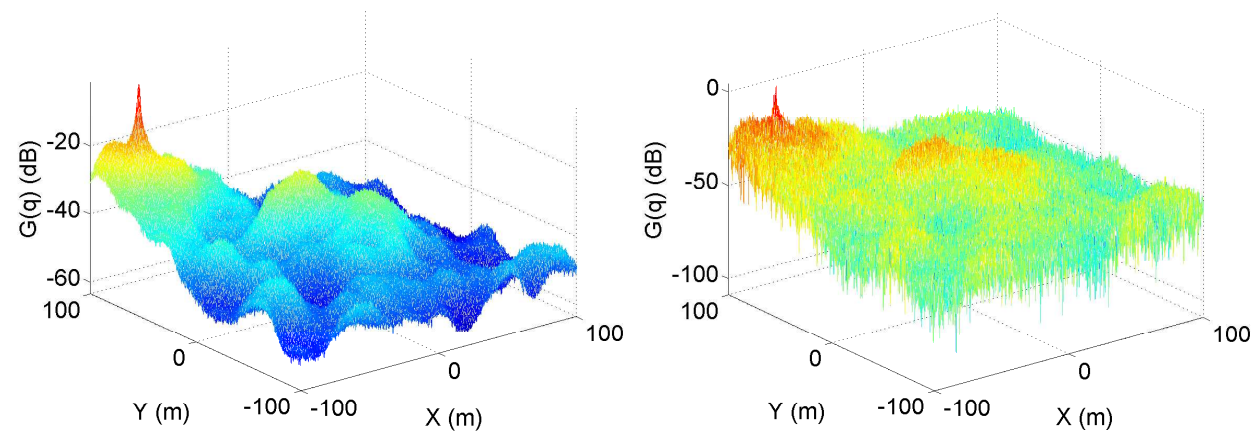

Fig. 9: Two sample channels with $\omega=0.8730 \mathrm{~dB}$ (left) and $\omega=5.0941 \mathrm{~dB}$ (right). The path loss and shadowing components of both channels are the same as in Fig. 2

per period for communication-intensive and communication-efficient cases respectively. The results for every $m$ are calculated by averaging over 500 different channels and sets of POIs. The POIs are distributed according to a uniform pdf over the workspace of Fig. 5. The channel in the $\mathrm{dB}$ domain is generated using a Gaussian distribution, with a mean equal to the path loss component of the channel of Fig. 3 and a standard deviation equal to $\vartheta=5 \mathrm{~dB}$. Also, in this example we set $P_{\ell, m}=P_{\ell, s}=P_{\ell}=0.1 \mathrm{~mW}, \rho=1$ and $\alpha=200$ for all the POIs. The rest of the parameters are the same as in Fig. 3. Note that we dropped the dependency of the parameters on $i$ and $k$ as there is only one mobile agent and the parameters are the same for all the POIs. Fig. 11 shows that the average minimum communication energy per period is an increasing function of the number of POIs in the communication-intensive case, as expected (the communication part of (32) is a linear function of $m_{k}$ ). However, it is a decreasing function of the number of POIs in the communication-efficient case. This is explained by the fact that by increasing the number of POIs in an environment, the chance of finding a higher channel power at one of the POIs increases. Also, it can be seen that overall the theoretical values provide a good approximation to the simulated ones.

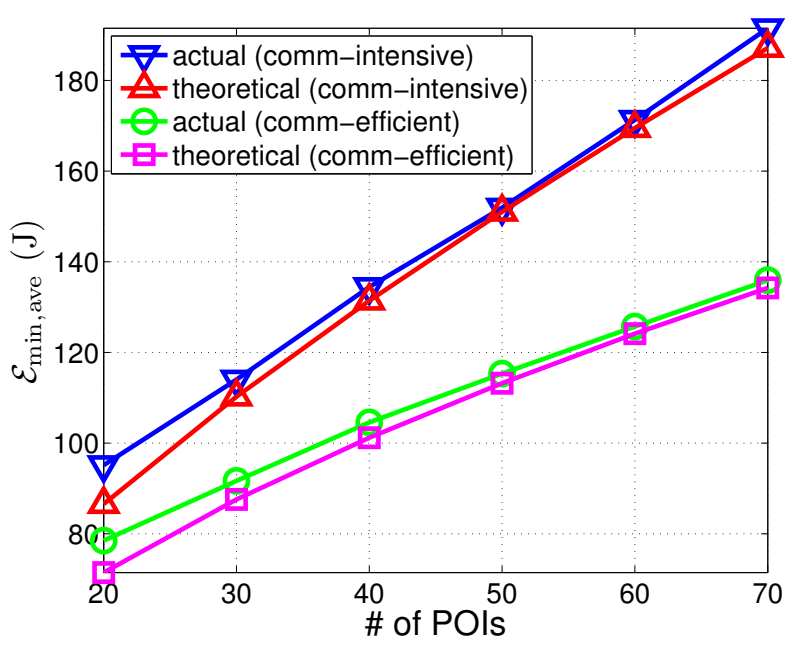

Fig. 10: The actual and theoretical average minimum total energy consumed in each period to cover a set of POIs, as a function of the number of POIs. 

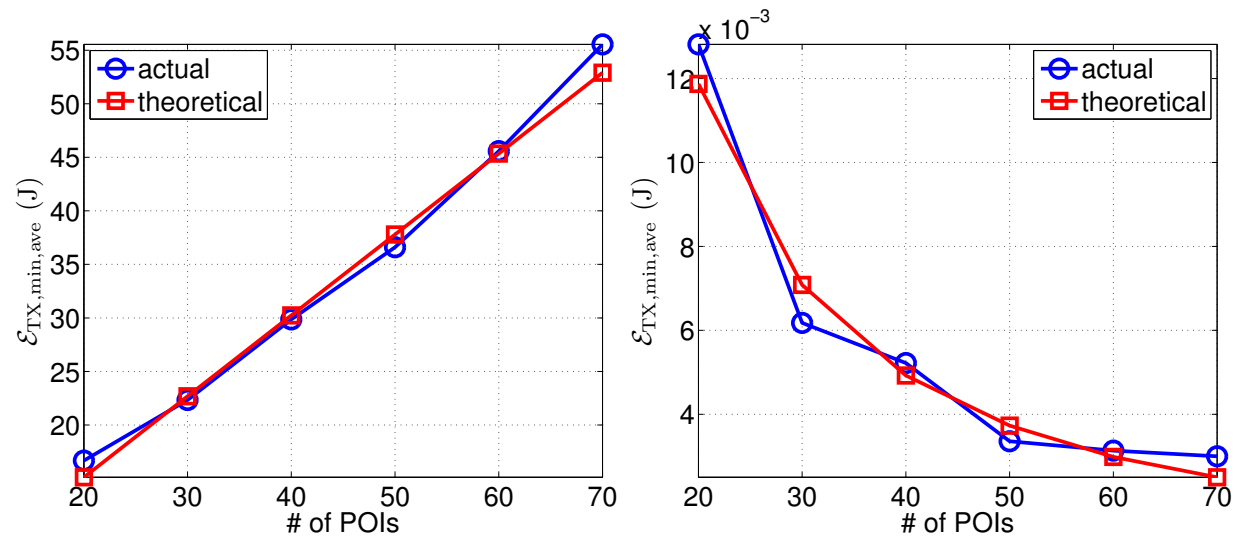

Fig. 11: The actual and theoretical average minimum communication energy consumed in each period to cover a set of POIs as a function of the number of POIs, in the communication-intensive (left) and communication-efficient (right) cases.

\section{CONCLUSIONS}

In this paper, we considered the problem of networked dynamic coverage of a number of POIs in a time-varying environment and in the presence of realistic fading channels. By a time-varying environment, we referred to an environment where a quantity of interest is constantly growing at certain rates at the POIs. We considered a linear dynamics for the time-variation of the quantity of interest at the POIs and a limited total energy budget for the mobile agents. We also considered the case where the sensing/actuation range of the mobile agents is small such that each agent is required to move to the position of each POI and stop there for some time to sense/service it. We then optimized motion (trajectories and stop times) and communication (transmission powers) of the mobile agents to minimize the total energy consumption of the mobile agents in each period, while guaranteeing that the quantity of interest at the POIs remains bounded, and the constraints on the connectivity of the mobile agents, the frequency of covering the POIs, and the total energy budget of the mobile agents are satisfied. We considered two variants of the problem: communication-intensive and communication-efficient. Communication-intensive case refers to the case where the mobile agents are required to be connected at all the POIs they visit, in order to send their collected information to the remote station in real-time. Communication-efficient case, on the other hand, refers to the case where the mobile agents are only required to connect to the remote station once along their trajectories, decreasing the communication burden considerably. In both cases, we showed how to optimally find the trajectories of the mobile agents, as well as their stop times and transmission powers at the POIs, using mixed-integer linear programs (MILPs). The properties of the optimal solutions of the MILPs, as well as their asymptotic properties, were also characterized mathematically.

Through theoretical analysis and simulation results, we showed that our proposed framework enables networked dynamic coverage of time-varying environments in the presence of realistic fading channels, which is not possible using the previous methods in the literature.

\section{APPENDIX}

\section{A. PROBABILISTIC ASSESSMENT OF THE SPATIAL VARIATIONS OF A WIRELESS CHANNEL}

Our proposed probabilistic channel assessment framework in [Ghaffarkhah and Mostofi 2011; Mostofi et al. 2010] can be used to assess the spatial variations of the channel power at the 
POIs based on a small number of channel power measurements in the same environment. It also provides a mathematical characterization of the channel assessment uncertainty (how much one can trust the channel assessment).

Let us start with probabilistic modeling of wireless channels. As shown in the communication literature [Goldsmith 2005], the channel power $G(q)$ can be probabilistically modeled as a multi-scale non-stationary random process, with three major dynamics: path loss, shadowing and multipath fading. We then have the following characterization for $G(q)$ (in $\mathrm{dB}$ ) using a 2D non-stationary random field model that characterizes all three dynamics of the channel [Goldsmith 2005]: $G_{\mathrm{dB}}(q)=K_{\mathrm{dB}}-10 n_{\mathrm{PL}} \log _{10}(\mathrm{~d}(q))+G_{\mathrm{SH}}(q)+G_{\mathrm{MP}}(q)$, where $G_{\mathrm{dB}}(q)=10 \log _{10}(G(q)), \mathrm{d}(q)$ is the Euclidean distance from $q \in \mathcal{W}$ to the remote station, $K_{\mathrm{dB}}$ and $n_{\mathrm{PL}}$ are path loss parameters, and $G_{\mathrm{SH}}(q)$ and $G_{\mathrm{MP}}(q)$ are independent random variables representing the effects of shadowing and multipath fading in $\mathrm{dB}$ respectively. The distributions of $G_{\mathrm{SH}}(q)$ and $G_{\mathrm{MP}}(q)$, as well as their spatial correlations, are typically given by empirical channel models. For instance, a lognormal distribution, with an exponential correlation, is a good fit for the distribution of $G_{\mathrm{SH}}(q)$ in linear domain. Nakagami, Rician, Rayleigh and lognormal distributions are also proven to match the distribution of $G_{\mathrm{MP}}(q)$ in linear domain. For more details on wireless channel modeling, see [Goldsmith 2005; Ghaffarkhah and Mostofi 2011; Mostofi et al. 2010].

Let $Q_{\mathrm{ch}}=\left[q_{\mathrm{ch}, 1}^{\mathrm{T}}, \cdots, q_{\mathrm{ch}, l}^{\mathrm{T}}\right]^{\mathrm{T}}$ denote the stacked vector of the positions corresponding to $l$ channel power measurements available. These measurements could be gathered through an offline survey of the channel at positions that are generally different from the positions of the POIs. Based on the probabilistic model for $G(q)$, the stacked vector of the received channel power measurements in $\mathrm{dB}$ can then be expressed by $Y_{\mathrm{ch}}=H\left(Q_{\mathrm{ch}}\right) \theta+\Xi_{\mathrm{ch}}+\Lambda_{\mathrm{ch}}$, where $H\left(Q_{\mathrm{ch}}\right)=\left[h\left(q_{\mathrm{ch}, 1}\right), \cdots, h\left(q_{\mathrm{ch}, l}\right)\right]^{\mathrm{T}}, h(q)=\left[1,-10 \log _{10}(\mathrm{~d}(q))\right]^{\mathrm{T}}$, for any $q \in \mathcal{W}, \theta=$ $\left[\begin{array}{ll}K_{\mathrm{dB}} & n_{\mathrm{PL}}\end{array}\right]^{\mathrm{T}}$ is the vector of path loss parameters, $\Xi_{\mathrm{ch}}=\left[G_{\mathrm{SH}}\left(q_{\mathrm{ch}, 1}\right), \cdots, G_{\mathrm{SH}}\left(q_{\mathrm{ch}, l}\right)\right]^{\mathrm{T}}$ and $\Lambda_{\mathrm{ch}}=\left[G_{\mathrm{MP}}\left(q_{\mathrm{ch}, 1}\right), \cdots, G_{\mathrm{MP}}\left(q_{\mathrm{ch}, l}\right)\right]^{\mathrm{T}}$. Based on the commonly used lognormal distribution for shadow fading and its reported exponential spatial correlation [Ghaffarkhah and Mostofi 2011; Mostofi et al. 2010], $\Xi_{\mathrm{ch}}$ is a zero-mean Gaussian random vector with the covariance matrix $R\left(Q_{\mathrm{ch}}\right)$, where $\left[R\left(Q_{\mathrm{ch}}\right)\right]_{i, j}=\vartheta^{2} \exp \left(-\frac{\left\|q_{\mathrm{ch}, i}-q_{\mathrm{ch}, j}\right\|}{\beta}\right)$, for $1 \leq i, j \leq l$, with $\vartheta^{2}$ and $\beta$ denoting the variance of the shadow fading component in $\mathrm{dB}$ and its decorrelation distance respectively. As for multipath fading, we assume uncorrelated lognormal distribution that results in a zero-mean Gaussian distribution with covariance matrix $\omega^{2} I_{l}$ for $\Lambda_{\text {ch }}$. Here, $\omega^{2}$ is the power of the multipath fading component (in $\mathrm{dB}$ ) and $I_{l}$ is the $l$-dimensional identity matrix. For a detailed discussion about validation of this model using real channel measurement, readers are referred to [Gonzalez-Ruiz et al. 2011; Goldsmith 2005].

As we proved in [Ghaffarkhah and Mostofi 2011; Mostofi et al. 2010], conditioned on the channel measurements and parameters $\theta, \vartheta, \beta$ and $\omega$, the assessment of the channel at an unvisited position $q \in \mathcal{W}$ is given by a Gaussian distribution with mean $\hat{G}_{\mathrm{dB}}(q)$ and variance $\sigma^{2}(q)$. We then have

$$
\begin{aligned}
\hat{G}_{\mathrm{dB}}(q) & =h^{\mathrm{T}}(q) \theta+\varphi^{\mathrm{T}}\left(q, Q_{\mathrm{ch}}\right) U^{-1}\left(Q_{\mathrm{ch}}\right)\left(Y_{\mathrm{ch}}-H\left(Q_{\mathrm{ch}}\right) \theta\right) \\
\sigma^{2}(q) & =\vartheta^{2}+\omega^{2}-\varphi^{\mathrm{T}}\left(q, Q_{\mathrm{ch}}\right) U^{-1}\left(Q_{\mathrm{ch}}\right) \varphi\left(q, Q_{\mathrm{ch}}\right)
\end{aligned}
$$

where $\varphi\left(q, Q_{\mathrm{ch}}\right)=\left[\vartheta^{2} e^{-\left\|q-q_{\mathrm{ch}, 1}\right\| / \beta}, \cdots, \vartheta^{2} e^{-\left\|q-q_{\mathrm{ch}, l}\right\| / \beta}\right]^{\mathrm{T}}$ and $U\left(Q_{\mathrm{ch}}\right)=R\left(Q_{\mathrm{ch}}\right)+\omega^{2} I_{l}$. Note that to assess the channel, the channel parameters $\theta, \vartheta, \beta$ and $\omega$ also need to be estimated. We, however, skip the details of the estimation of the underlying parameters and refer readers to our previous work in [Ghaffarkhah and Mostofi 2011; Mostofi et al. 2010] for more details. 


\section{B. PROOF OF THEOREM 3.1}

Consider (11), where a partition $\left\{\mathcal{V}_{k}\right\}_{k=1}^{n}$ is given. In this case the problem becomes decoupled and can be solved individually for each mobile agent. It can immediately be seen that the optimal transmission power for each mobile agent $k$ at the $i$ th POI is the minimum transmission power required to guarantee the probability of connectivity at the POI is no smaller than $\chi$, i.e., $P_{\mathrm{TX}, i, k}^{*}=P_{\mathrm{TX}, \min }\left(q_{i}, \chi\right)$. Thus, the first set of conditions for feasibility of the problem are $P_{\mathrm{TX}, \min }\left(q_{i}, \chi\right) \leq P_{\mathrm{TX}, \max , k}$, for $i \in \mathcal{V}_{k}$ and $k=1, \cdots, n$. Moreover, for any set of stop times $t_{i, k}$, for $i \in \mathcal{V}_{k}$, the total energy and the period are increasing functions of $\mathrm{d}\left(\mathcal{H}_{k}\right)$ and decreasing functions of $v_{k}$. Then, to obtain the minimum total energy, the optimal cycle is the one with the minimum total length, i.e., the minimum-length Hamiltonian cycle $\mathcal{H}_{k}^{*}$, and the optimal velocity is the maximum possible velocity, i.e., $v_{\max , k}$.

Let us replace $\mathrm{d}\left(\mathcal{H}_{k}\right)$ with $\mathrm{d}\left(\mathcal{H}_{k}^{*}\right)$ and $v_{k}$ with $v_{\max , k}$ in (11). Also, let us define $\overline{\mathcal{E}}_{\max , k} \triangleq$ $\mathcal{E}_{\max , k}-t_{c} \sum_{i \in \mathcal{V}_{k}} P_{\mathrm{TX}, \min }\left(q_{i}, \chi\right)$. Then, the optimal stop times for each mobile agent $k$ are given by the solution of the following optimization problem, provided that the optimal stop times are all positive:

$$
\begin{aligned}
& \min \sum_{i \in \mathcal{V}_{k}} t_{i, k}, \\
& \text { s.t. } \\
& \text { 1) } \alpha_{i, k} t_{i, k}-\rho_{i}\left(\sum_{j \in \mathcal{V}_{k}} t_{j, k}+\frac{\mathrm{d}\left(\mathcal{H}_{k}^{*}\right)}{v_{\max , k}}\right) \geq \Delta_{k}, \quad \forall i \in \mathcal{V}_{k}, \\
& \text { 2) } \sum_{i \in \mathcal{V}_{k}} t_{i, k}+\frac{\mathrm{d}\left(\mathcal{H}_{k}^{*}\right)}{v_{\max , k}} \leq T_{\max }, \\
& \text { 3) } P_{\ell, s, k} \sum_{i \in \mathcal{V}_{k}} t_{i, k}+\left(\frac{P_{\ell, m, k}}{v_{\max , k}}+w_{k}\right) \mathrm{d}\left(\mathcal{H}_{k}^{*}\right) \leq \overline{\mathcal{E}}_{\max , k} .
\end{aligned}
$$

The optimal solution of this linear program satisfies the Karush-Kuhn-Tucker (KKT) conditions. The Lagrangian of the problem is given by

$$
\begin{gathered}
L_{k}=\sum_{i \in \mathcal{V}_{k}} t_{i, k}-\sum_{i \in \mathcal{V}_{k}} \mu_{i, k}\left(\alpha_{i, k} t_{i, k}-\rho_{i} \sum_{j \in \mathcal{V}_{k}} t_{j, k}-\rho_{i} \frac{\mathrm{d}\left(\mathcal{H}_{k}^{*}\right)}{v_{\max , k}}-\Delta_{k}\right)+ \\
\gamma_{k}\left(\sum_{i \in \mathcal{V}_{k}} t_{i, k}+\frac{\mathrm{d}\left(\mathcal{H}_{k}^{*}\right)}{v_{\max , k}}-T_{\max }\right)+\nu_{k}\left(P_{\ell, s, k} \sum_{i \in \mathcal{V}_{k}} t_{i, k}+\frac{P_{\ell, m, k} \mathrm{~d}\left(\mathcal{H}_{k}^{*}\right)}{v_{\max , k}}+w_{k} \mathrm{~d}\left(\mathcal{H}_{k}^{*}\right)-\overline{\mathcal{E}}_{\max , k}\right),
\end{gathered}
$$

where $\mu_{i, k}, \gamma_{k}$ and $\nu_{k}$ are the Lagrange multipliers. The KKT conditions are then as follows:

$$
\begin{aligned}
& \text { 1) } \frac{\partial L_{k}}{\partial t_{i, k}}=1-\alpha_{i, k} \mu_{i, k}+\sum_{j \in \mathcal{V}_{k}} \mu_{j, k} \rho_{j}+\gamma_{k}+\nu_{k} P_{\ell, s, k}=0, \quad \forall i \in \mathcal{V}_{k}, \\
& \text { 2) } \mu_{i, k}\left(\alpha_{i, k} t_{i, k}-\rho_{i} \sum_{j \in \mathcal{V}_{k}} t_{j, k}-\rho_{i} \frac{\mathrm{d}\left(\mathcal{H}_{k}^{*}\right)}{v_{\max , k}}-\Delta_{k}\right)=0, \quad \forall i \in \mathcal{V}_{k}, \\
& \text { 3) } \gamma_{k}\left(\sum_{i \in \mathcal{V}_{k}} t_{i, k}+\frac{\mathrm{d}\left(\mathcal{H}_{k}^{*}\right)}{v_{\max , k}}-T_{\max }\right)=0 \\
& \text { 4) } \nu_{k}\left(P_{\ell, s, k} \sum_{i \in \mathcal{V}_{k}} t_{i, k}+\frac{P_{\ell, m, k} \mathrm{~d}\left(\mathcal{H}_{k}^{*}\right)}{v_{\max , k}}+w_{k} \mathrm{~d}\left(\mathcal{H}_{k}^{*}\right)-\overline{\mathcal{E}}_{\max , k}\right)=0, \\
& \text { 5) } \alpha_{i, k} t_{i, k}-\rho_{i} \sum_{j \in \mathcal{V}_{k}} t_{j, k}-\rho_{i} \frac{\mathrm{d}\left(\mathcal{H}_{k}^{*}\right)}{v_{\max , k}}-\Delta_{k} \geq 0 \\
& \text { 6) } \sum_{i \in \mathcal{V}_{k}} t_{i, k}+\frac{\mathrm{d}\left(\mathcal{H}_{k}^{*}\right)}{v_{\max , k}}-T_{\max } \leq 0 \\
& \text { 7) } P_{\ell, s, k} \sum_{i \in \mathcal{V}_{k}} t_{i, k}+\frac{P_{\ell, m, k} \mathrm{~d}\left(\mathcal{H}_{k}^{*}\right)}{v_{\max , k}}+w_{k} \mathrm{~d}\left(\mathcal{H}_{k}^{*}\right)-\overline{\mathcal{E}}_{\max , k} \leq 0, \\
& \text { 8) } \mu_{i, k} \geq 0, \forall i \in \mathcal{V}_{k}, \gamma_{k} \geq 0, \nu_{k} \geq 0 .
\end{aligned}
$$

From the first set of conditions in (48), we conclude that $\mu_{i, k}>0$, for $i \in \mathcal{V}_{k}$, which results in the following optimality conditions: $\alpha_{i, k} t_{i, k}-\rho_{i} \sum_{j \in \mathcal{V}_{k}} t_{j, k}-\rho_{i} \frac{\mathrm{d}\left(\mathcal{H}_{k}^{*}\right)}{v_{\max , k}}=\Delta_{k}$, for $i \in \mathcal{V}_{k}$. 
By solving this set of equations, we get the following for the optimal stop times:

$$
\begin{aligned}
t_{i, k}^{*} & =\Delta_{k}\left(\frac{1}{\alpha_{i, k}}+\frac{\rho_{i} \eta_{k}}{\alpha_{i, k} \phi_{k}}\right)+\frac{\rho_{i}}{\alpha_{i, k} \phi_{k}} \frac{\mathrm{d}\left(\mathcal{H}_{k}^{*}\right)}{v_{\max , k}}, \quad \forall i \in \mathcal{V}_{k}, \\
\sum_{i \in \mathcal{V}_{k}} t_{i, k}^{*} & =\Delta_{k} \frac{\eta_{k}}{\phi_{k}}+\frac{\mathrm{d}\left(\mathcal{H}_{k}^{*}\right)}{v_{\max , k}} \frac{1-\phi_{k}}{\phi_{k}},
\end{aligned}
$$

where $\phi_{k} \triangleq 1-\sum_{i \in \mathcal{V}_{k}} \frac{\rho_{i}}{\alpha_{i, k}}$ and $\eta_{k} \triangleq \sum_{i \in \mathcal{V}_{k}} \frac{1}{\alpha_{i, k}}$. It can be seen that the optimal stop times are all positive when $\sum_{i \in \mathcal{V}_{k}} \frac{\rho_{i}}{\alpha_{i, k}}<1$, for $k=1, \cdots, n$, which form the second set of conditions for the feasibility of the optimization problem. The third set of such conditions are also given as follows:

$$
\begin{aligned}
& P_{\ell, s, k}\left(\Delta_{k} \frac{\eta_{k}}{\phi_{k}}+\frac{\mathrm{d}\left(\mathcal{H}_{k}^{*}\right)}{v_{\max , k}} \frac{1-\phi_{k}}{\phi_{k}}\right)+\left(\frac{P_{\ell, m, k}}{v_{\max , k}}+w_{k}\right) \mathrm{d}\left(\mathcal{H}_{k}^{*}\right) \leq \overline{\mathcal{E}}_{\max , k}, \\
& \Delta_{k} \frac{\eta_{k}}{\phi_{k}}+\frac{\mathrm{d}\left(\mathcal{H}_{k}^{*}\right)}{v_{\max , k}} \frac{1}{\phi_{k}} \leq T_{\max , k} .
\end{aligned}
$$

After combining these two constraints, we then obtain the third set of feasibility conditions in part 1 of Theorem 3.1. The maximum stability margin is also the maximum $\Delta_{k}$ that satisfies (50), which can be shown to be the same as $\Delta_{\max , k}$ in part 2 of Theorem 3.1.

\section{REFERENCES}

IBM ILOG CPLEX Optimizer, Available: http://www-01.ibm.com/software/integration/optimization/ cplex-optimizer/.

SAS/OR Software, http://www.sas.com/technologies/analytics/optimization/or/.

Acar, E. U. And Choset, H. 2002. Sensor-based Coverage of Unknown Environments: Incremental Construction of Morse Decompositions. The International Journal of Robotics Research 21, 4, 345-366.

Acar, E. U., Choset, H., And Ji, Y. L. 2006. Sensor-based coverage with extended range detectors. IEEE Transactions on Robotics 22, 1, 189-198.

Agmon, N., Urieli, D., And Stone, P. 2011. Multiagent Patrol Generalized to Complex Environmental Conditions. In Proceedings of the Twenty-Fifth Conference on Artificial Intelligence.

Bektas, T. 2006. The multiple traveling salesman problem: an overview of formulations and solution procedures. Omega 34, 3, 209-219.

Bullo, F., Frazzoli, E., Pavone, M., Savla, K., And Smith, S. L. 2011. Dynamic vehicle routing for robotic systems. Proceedings of the IEEE 99, 9, 1482-1504.

Chevaleyre, Y. 2004. Theoretical analysis of the multi-agent patrolling problem. In Proceeding of IEEE/WIC/ACM International Conference on Intelligent Agent Technology (IAT). 302-308.

Choset, H. 2001. Coverage for robotics - A survey of recent results. Annals of Mathematics and Artificial Intelligence 31, 113-126.

Elmaliach, Y., Agmon, N., and KaminkA, G. 2009. Multi-robot area patrol under frequency constraints. Annals of Mathematics and Artificial Intelligence 57, 293-320.

Ghaffarkhah, A. And Mostofi, Y. 2011. Communication-Aware Motion Planning in Mobile Networks. IEEE Transactions on Automatic Control 56, 10, 2478-2485.

Ghaffarkhah, A., Yan, Y., and Mostofi, Y. 2011. Dynamic Coverage of Time-Varying Environments Using a Mobile Robot - a Communication-Aware Perspective. In Proceedings of IEEE Globecom International Workshop on Wireless Networking for Unmanned Autonomous Vehicles (Wi-UAV). Houston, TX.

Goldsmith, A. 2005. Wireless Communications. Cambridge University Press.

Gonzalez-Ruiz, A., Ghaffarkhah, A., And Mostofi, Y. 2011. A Comprehensive Overview and Characterization of Wireless Channels for Networked Robotic and Control Systems. Journal of Robotics, 2011.

Grocholsky, B., Keller, J., Kumar, V., and Pappas, G. 2006. Cooperative Air and Ground Surveillance. IEEE Robotics and Automation Magazine 13, 3, 16-25.

Gutin, G. And Punnen, A. P. 2004. The Traveling Salesman Problem and Its Variations (Combinatorial Optimization). Kluwer Academic Press. 
Hokayem, P. F., Stipanovic, D., And Spong, M. W. 2007. On persistent coverage control. In Proceedings of 46th IEEE Conference on Decision and Control (CDC). 6130-6135.

Larson, R. C. And Odoni, A. R. Urban Operations Research. Prentice-Hall, Englewood Cliffs, NJ.

Li, M., Cheng, W., Liu, K., He, Y., Li, X., and Liao, X. 2011. Sweep Coverage with Mobile Sensors. IEEE Transactions on Mobile Computing 10, 11, 1534-1545.

Machado, A., Ramalho, G., Zucker, J. D., and Drogoul, A. 2003. Multi-agent patrolling: an empirical analysis of alternative architectures. In Proceedings of the 3rd international conference on Multi-agentbased simulation II. MABS'02. Springer-Verlag, Berlin, Heidelberg, 155-170.

Mei, Y., Lu, Y., Hu, Y. C., And Lee, C. S. G. 2005. A Case Study of Mobile Robots Energy Consumption and Conservation Techniques. In Proceeding of the 12th International Conference on Advanced Robotics (ICAR). 492-497.

Mei, Y., Lu, Y., Hu, Y. C., And Lee, C. S. G. 2006. Deployment of Mobile Robots With Energy and Timing Constraints. IEEE Transactions on Robotics 22, 3, 507-522.

Mostofi, Y., Gonzalez-Ruiz, A., Ghaffarkhah, A., And Li, D. 2009. Characterization and Modeling of Wireless Channels for Networked Robotic and Control Systems - A Comprehensive Overview. In Proceedings of 2009 IEEE/RSJ International Conference on Intelligent Robots and Systems (IROS). St. Louis, MO, 4849-4854.

Mostofi, Y., Malmirchegini, M., and Ghaffarkhah, A. 2010. Estimation of Communication Signal Strength in Robotic Networks. In Proceedings of IEEE International Conference on Robotics and Automation (ICRA). Anchorage, AK, 1946-1951.

NA, B. 2007. Heurisic approaches for no-depot k-traveling salesmen problem with a minmax objective. M.S. thesis, Texas A\&M University.

Shapiro, A., Dentcheva, D., And Ruszczynski, A. 2009. Lectures on Stochastic Programming: Modeling and Theory. SIAM.

Sinopoli, B., Schenato, L., Franceschetti, M., Poolla, K., Jordan, M. I., and Sastry, S. S. 2004. Kalman filtering with intermittent observations. IEEE Transactions on Automatic Control 49, 9, 14531464.

Smith, R. N., Schwager, M., Smith, S. L., Jones, B. H., Rus, D., and Sukhatme, G. S. 2011. Persistent Ocean Monitoring with Underwater Gliders: Adapting Sampling Resolution. Journal of Field Robotics 28, 5, 714-741.

Smith, S. L., Schwager, M., And Rus, D. 2012. Persistent Robotic Tasks: Monitoring and Sweeping in Changing Environments. IEEE Transactions on Robotics 28, 2, 410-426.

Son, D., Krishnamachari, B., and Heidemann, J. 2006. Experimental Study of Concurrent Transmission in Wireless Sensor Networks. In Proc. of the 4th Intl. Conf. on Embedded Networked Sensor Systems. $237-250$.

Wang, Y. AND Hussein, I. 2010. Awareness Coverage Control Over Large-Scale Domains With Intermittent Communications. IEEE Transactions on Automatic Control 55, 8, 1850-1859. 